Impacto del Plan Nacer sobre la Mortalidad Infantil en Argentina

Santiago Garriga

Tesis de Maestría

Maestría en Economía

Universidad Nacional de La Plata

Director: Guido Porto

Co-Directora: Natalia Porto

Códigos JEL: C33, I12, I38 


\title{
Impacto del Plan Nacer sobre la Mortalidad Infantil en Argentina
}

\author{
Santiago Garriga*
}

Agosto 2012

\section{Resumen}

El Plan Nacer es un programa del Ministerio de Salud de la Nación Argentina que invierte recursos con el objetivo de mejorar la cobertura y calidad de los servicios de salud en niños menores de seis años, mujeres embarazadas y puérperas que no poseen obra social. La novedad del mismo radica en la generación de un seguro público de salud para la población materno-infantil sin cobertura social y en la aplicación de un modelo de financiamiento basado en resultados. Este trabajo investiga los efectos del Plan Nacer sobre la mortalidad infantil en la Argentina siguiendo un enfoque no-experimental, basándose particularmente en la metodología de diferencias en diferencias, que consiste en la comparación de condiciones sanitarias entre poblaciones inscriptas y no inscriptas al programa, antes y después de su implementación. La fuente principal de variación es la participación en el programa a nivel departamental. El análisis de impacto realizado considera el caso de un tratamiento en donde las unidades bajo programa están sujetas a distintos grados de exposición al mismo. Los resultados encontrados indican que el Plan Nacer genera una reducción de la tasa de mortalidad infantil. El resultado es ambiguo si se divide a la tasa de mortalidad infantil entre la tasa de mortalidad neonatal, y la tasa de mortalidad post-neonatal: mientras que el Plan tiene un impacto negativo sobre la segunda, no ocurre lo mismo con la primera. Además no parecería existir un efecto del programa sobre la tasa de mortalidad materna.

JEL: C33, I12, I38

Palabras clave: mortalidad infantil, evaluación de impacto, diferencias en diferencias, Plan Nacer.

* Facultad de Ciencias Económicas - Universidad Nacional de La Plata. Este trabajo constituye la Tesis de Maestría en Economía de la Universidad Nacional de La Plata Argentina, realizada bajo la dirección de Dr. Guido Porto y la codirección de la Dra. Natalia Porto. Mi agradecimiento a ellos por su ayuda, predisposición, apoyo y compromiso durante todo el proceso de tesis. Los errores u omisiones son total responsabilidad del autor. 


\section{Introducción}

El Plan Nacer es un programa del Ministerio de Salud de la Nación Argentina que, reconociendo la organización federal del país, invierte recursos con el objetivo de mejorar la cobertura de salud y la calidad de atención de las mujeres embarazadas, puérperas y de los niños menores de 6 años sin obra social. ${ }^{1}$ Específicamente, se propone reducir la morbilidad y mortalidad materno-infantil, financiando un conjunto de prestaciones seleccionadas por su alto impacto y su costo efectividad que promueven la atención y el cuidado de la madre y el niño.

En él intervienen múltiples actores entre los que se destacan la nación, las provincias y los establecimientos de salud. Existen dos tipos de relaciones que determinan los flujos de fondos en la estrategia de financiamiento implementada. La primera relación está dada entre el gobierno nacional y las provincias, donde la nación transfiere recursos a éstas en función de la nominalización de la población elegible y el cumplimiento de una serie de metas sanitarias. Por otro lado, los seguros provinciales, que tienen a su cargo la implementación del Plan Nacer en sus territorios, transfieren fondos a los establecimientos de salud en función de un conjunto de prácticas priorizadas que brindan a su población a cargo, agrupadas en el Nomenclador Único.

Este trabajo investiga los efectos del Plan Nacer sobre la mortalidad infantil en Argentina definida como las defunciones de personas menores a un año. La evaluación de programas es un componente importante de los mismos, ya que aporta evidencia de su efectividad, ayuda a mejorar su diseño, y permite su sostenimiento o no, al momento de rendir cuentas por el uso de los recursos empleados. Específicamente, la evaluación de impacto permite analizar los efectos causales de un programa sobre una serie de variables de resultado escogidas. Este estudio realiza una evaluación de impacto siguiendo un enfoque no-experimental, basándose particularmente en la metodología de diferencias en diferencias, que consiste en la comparación de condiciones sanitarias entre poblaciones bajo programa y sin el mismo, antes y después de su implementación. El análisis de impacto realizado considera el caso de un tratamiento en donde las unidades bajo programa están sujetas a distintos grados de exposición al mismo. La fuente principal de variación es la participación en el programa a nivel departamental. Esta variación a nivel departamental existe solamente por un plazo limitado, en particular, entre el comienzo del programa en las provincias del noroeste y del noreste argentino en el año 2005 hasta la ampliación al resto de las provincias a fines del año 2007.

La idea subyacente es que el Plan Nacer genera, mediante normativas y contratos de gestión, recursos en función de resultados. Esto permite una correcta asignación de incentivos entre

\footnotetext{
${ }^{1}$ El puerperio es el período que sigue inmediatamente al parto y que se extiende por aproximadamente 45 días hasta que el cuerpo materno vuelve a las condiciones pre-gestacionales, perdiendo las características adquiridas durante el embarazo.
} 
los distintos actores del programa lo que se traduce, en última instancia, en una mejora en el acceso a los servicios de salud y la calidad de las prestaciones realizadas. Consecuentemente, es de esperarse que la población bajo programa mejore su condición sanitaria y que esto se refleje en distintas variables de resultados en salud, entre las que naturalmente surge la tasa de mortalidad infantil como la más relevante. Como esta última puede depender, entre varias cosas, de la intensidad regional de los establecimientos de salud, es decir de la relación entre la cantidad de establecimientos y su población de referencia, por cuestiones que no necesariamente están relacionadas con el Plan Nacer, se realizan varios experimentos de falsificación. Estos experimentos consisten en explorar si la estrategia de identificación falla en intervenciones tipo placebo, por ejemplo examinando regiones no afectadas por el Plan Nacer o regiones afectadas por el Plan pero en momentos en los que éste no estaba vigente.

Esta investigación tiene, principalmente, dos motivaciones. La primera de ellas se relaciona con el estudio de la mortalidad infantil como indicador de bienestar social y consecuentemente con el análisis de su evolución y programas que busquen su alivio. El segundo componente del trabajo se relaciona con la importancia de realizar evaluaciones de programas o políticas sociales, por diversas razones, y particularmente con la realización de evaluaciones de impacto. La generación de evidencia acerca de qué es lo que funciona y que no, en términos de desarrollo humano, es un camino hacia la mejora en la efectividad de las políticas públicas. El análisis de impacto realizado no solo considera un "tratamiento" binario sino también el caso de un tratamiento en donde las unidades bajo programa están sujetas a distintos grados de exposición al mismo. Las evaluaciones que consideran este tipo de tratamientos, parecieran acercarse más a la realidad en términos del grado de exposición que reciben las unidades bajo tratamiento.

Para conocimiento del autor no existe al momento de realizarse el presente estudio otros que investiguen el impacto del Plan Nacer. La única excepción es el trabajo de Gertler, Martinez y Celhay (2011) quienes explotan las bases administrativas generadas en las provincias de Misiones y de Tucumán, y encuentran evidencia de fuertes impactos del Plan Nacer en el cuidado prenatal y del niño sano. ${ }^{2}$ En Misiones verifican que aumentan en 3 veces las consultas previas al parto generando mejores resultados en la salud del niño, y en Tucumán observan una significativa reducción en la probabilidad de muerte en los primeros siete días de vida entre los niños incluidos en el Plan Nacer, quienes a su vez reciben más controles. Los autores encuentran evidencia, para ambas provincias, de que las mujeres embarazadas son detectadas por el sistema de salud más tempranamente.

\footnotetext{
${ }^{2}$ Los registros administrativos en estas provincias incluyen atenciones prenatales, atención a partos, y cuidados del niño sano en Centros de Atención Primaria y Maternidades del sector público.
} 
Los resultados encontrados en el presente estudio muestran que el Plan Nacer genera una reducción de la tasa de mortalidad infantil. Más específicamente, el Plan mejora el acceso a los servicios de salud y la calidad de las prestaciones, lo que impacta sobre la tasa de mortalidad de los niños menores de un año. De todas formas, el resultado es ambiguo si se divide a la tasa de mortalidad infantil entre tasa de mortalidad neonatal, referida a las muertes de los menores de veintisiete días, y tasa de mortalidad post-neonatal, que ocurre entre los veintisiete días y el año de vida. Mientras que el Plan tiene un impacto sobre la segunda, no ocurre lo mismo con la primera. Además no pareciera existir un efecto del programa sobre la tasa de mortalidad materna.

El trabajo se organiza de la siguiente manera. En la sección 2 se enumeran y desarrollan las motivaciones que llevaron a realizar el estudio y se presenta una breve revisión de la literatura. En la sección 3 se detallan las principales características del Plan Nacer y se realiza un análisis exploratorio de la tasa de mortalidad infantil, desde su definición hasta su distribución en Argentina y el mundo. En la sección 4 se expone la metodología utilizada para la estimación, los distintos resultados obtenidos y los experimentos de falsificación de manera de robustecer el análisis. Por último, la sección 5 concluye con algunos comentarios finales.

\section{Motivación del Estudio}

La motivación del trabajo se basa en dos pilares fundamentales, siendo uno de ellos el estudio de la mortalidad infantil. En general, se reconoce a esta última como uno de los indicadores que mejor sintetiza las condiciones de salud de una población en particular, soslayando el hecho de que los niños son el grupo poblacional con menores defensas inmunológicas. Según Alderman y Behrman (2004), la tasa de mortalidad infantil es una medida del grado de supervivencia infantil y como tal, es considerada uno de los indicadores más robustos del bienestar de un país, ya que ilustra las circunstancias sociales, económicas y ambientales en las que los niños y la sociedad en general, viven. En ese sentido, la tasa de mortalidad infantil se muestra como un indicador clave del nivel de calidad de vida de una población en particular por lo que el estudio de sus determinantes, evolución y programas que busquen su alivio, resulta particularmente importante.

La utilización de la tasa de mortalidad infantil como un indicador relevante del bienestar de una sociedad data desde principios del siglo XX. Arthur Nesholme, un asesor de alto rango en materia de salud del Reino Unido, escribía a principios de siglo "La tasa de mortalidad infantil es el indicador más sensible del bienestar social y de administración sanitaria, especialmente bajo condiciones urbanas" (citado en Titmuss, 1943, p12). Más cerca en el tiempo, específicamente en el año 2000, la tasa de mortalidad infantil fue seleccionada como uno de los indicadores para lograr los Objetivos del Milenio fijados por los países miembros de las Naciones Unidas. Según el Informe 
de Desarrollo Humano elaborado por el Programa de las Naciones Unidas para el desarrollo (PNUD, 2005), la mortalidad infantil es el indicador que mejor capta las divergencias en materia de oportunidades de desarrollo humano.

Una referencia reciente aplicada al estudio de la mortalidad infantil en Argentina es el trabajo de Cruces, Glüzmann y López Calva (2011), que estudia los efectos de las crisis económicas sobre la mortalidad infantil y materna, sobre la proporción de nacidos vivos con bajo peso al nacer y sobre las tasas de asistencia escolar. En la misma línea, Bozzoli y Quintana-Domeque (2010) estudian el efecto de la crisis económica del 2001-2002 en Argentina sobre el peso al nacer.

El segundo componente central del trabajo se relaciona con la importancia de realizar evaluaciones de programas o políticas sociales. La evaluación aporta evidencia sobre la efectividad de las políticas públicas, algo particularmente relevante en un contexto de recursos limitados. Al mismo tiempo, brinda información que es utilizada luego para tomar decisiones, como por ejemplo para promover o expandir políticas con impactos positivos, o para reformular políticas con impactos negativos. En ese sentido, la evidencia obtenida puede utilizarse para sostener un programa por un período prolongado o para ampliar su escala. Asimismo, la evaluación ayuda a mejorar su diseño ya que, entre otras cosas, permite entender por qué funciona una política, cuáles son sus mecanismos y quiénes se benefician.

Existen diversos tipos de evaluación: la evaluación costo-beneficio y costo-efectividad, la evaluación de procesos, la evaluación cualitativa y la evaluación de impacto. Todos estos tipos de evaluaciones son herramientas complementarias entre sí. Específicamente el trabajo propone realizar una evaluación de impacto del programa, pero es importante destacar que las demás son también relevantes para realizar una evaluación integral de un programa de política pública. ${ }^{3}$ La evaluación de impacto mide la relación causal entre un programa o política y los resultados en los beneficiarios, comparando la situación de los beneficiarios en ausencia de la intervención (con grupos de comparación) con el estado observado de los beneficiarios (grupo de tratamiento), para determinar el efecto (impacto) de la intervención en los resultados intermedios o finales.

La generación de evidencia acerca de qué es lo que funciona y que no en términos de desarrollo humano, es un camino hacia el perfeccionamiento en la efectividad de las políticas públicas que buscan mejorar el bienestar de una sociedad en particular. En el año 2004 el Centro

\footnotetext{
${ }^{3}$ Feinstein (2007) plantea que, más allá de que el interés esté puesto sobre el impacto de los programas sociales y por ende, en las evaluaciones de impacto, existen situaciones en las cuales no es posible aislar el efecto atribuible a una intervención en particular, por lo tanto es importante complementar el análisis de impacto con un análisis de procesos. En ese sentido, sostiene que existe una tendencia creciente en valorizar resultados en desmedro de los procesos, sin embargo las dificultades propias de la atribución de resultados, vuelve relevante estudiar los procesos asociados a una intervención ya aportan cierta claridad a dicha atribución. Puede pensarse entonces a las evaluaciones de procesos como complementarias a las de resultados.
} 
para el Desarrollo Global (CGD por sus siglas en inglés), crea el Evaluation Gap Working Group (Grupo de Trabajo para la Brecha de la Evaluación) con el objetivo de investigar el estado de generación de evidencia acerca de la efectividad de los programas sociales e iniciativas para el desarrollo, y de realizar recomendaciones o sugerencias para estimular y mejorar las evaluaciones. El mencionado grupo elaboró un informe titulado "When will we ever learn? Improving lives through impact evaluation” (2006), en donde se destaca el hecho de que es muy pobre la evidencia que existe acerca de la efectividad de los programas sociales más allá de que se destina una cantidad de recursos considerable para su ejecución. Los autores resaltan la necesidad de abordar la brecha de evaluación a través de la generación de este conocimiento y la divulgación de su uso y aplicación.

Las iniciativas generadas en México para estimular una cultura de evaluación han colocado al país en la vanguardia de estos temas. El dictado de la Ley General de Desarrollo social (2001) que establece la obligatoriedad de la evaluación de programas sociales, y la creación del Consejo Nacional de Evaluación de la política de Desarrollo social (CONEVAL) como institución responsable de verificar la consistencia y el cumplimiento de los objetivos de la política social, así como de coordinar y ejecutar las evaluaciones a los programas sociales, son una muestra clara de ello. Más allá del caso mexicano, el resto de los países latinoamericanos carece de una cultura de evaluación lo suficientemente rigurosa; sin embargo, parecería existir una tendencia creciente a realizar este tipo de estudios. Dos referencias interesantes que analizan el estado de las evaluaciones de impacto en Latinoamérica son Bouillon y Tejerina (2007), y Alzúa, Djebbari y Valdivia (2012). Para el caso de Argentina, una referencia obligada vinculada con la mortalidad infantil es el trabajo de Galiani, Gertler y Schargrodsky (2005) quienes estudian el impacto de la privatización en la provisión de agua sobre la mortalidad infantil. Utilizando la variación temporal y espacial del proceso mismo de privatización, encuentran que en las áreas privatizadas disminuye la mortalidad infantil y que esto se da con mayor fuerza en las áreas más pobres.

Para realizar un evaluación de impacto de calidad es necesario, según Gertler, Martinez, Premand, Rawlings y Vermeersch (2010), que el programa a evaluar cumpla una serie de condiciones deseables, entre ellas, que sea innovador, replicable, estratégicamente pertinente, no comprobado, e influyente. Innovador en el sentido de que sea reciente y que ponga a prueba un nuevo enfoque. Que el programa sea replicable se refiere a que pueda ampliarse y aplicarse a una situación diferente. Estratégicamente pertinente, alude a que debe requerir una cantidad de recursos considerable (o generar un ahorro de recursos considerable) o cubrir a un gran número de personas. Que no haya sido comprobado quiere decir que debe saberse muy poco sobre la efectividad del 
mismo en general. Por último, que sea influyente implica que los resultados deben ser útiles en el proceso de toma de decisiones sobre políticas.

El programa que será evaluado cumple todas las condiciones citadas en el párrafo anterior. Es innovador porque inaugura una nueva forma de buscar resultados en la implementación de políticas sociales en el sector salud. El Plan Nacer realiza transferencias en función del cumplimiento de resultados explícitamente consensuados y formalizados que son evaluados por auditorías externas e independientes. Es replicable y puede aplicarse a otras situaciones, de hecho se inició en las regiones de NOA y NEA y años más tarde se amplió al resto del país. ${ }^{4}$ Obviamente el programa es estratégicamente pertinente porque está destinado a mejorar las condiciones de salud de las personas sin cobertura explícita lo que hace que el número de beneficiarios efectivos y/o potenciales sea grande. Además, tal como se mencionó en la sección anterior, se trata de un programa poco comprobado, siendo el único trabajo que realizó una evaluación de impacto del Plan Nacer, el de Gertler et al. (2011). Por último, la evaluación del programa pretende tener cierto grado de influencia sobre aquellos que se encuentran en la órbita de la toma de decisiones.

El análisis de impacto realizado no solo considera un "tratamiento" binario sino también distintos grados de exposición al programa de las unidades tratadas. En los últimos años, la literatura de evaluación de programas sociales, ha mutado de un enfoque de tratamiento binario a tratamientos más heterogéneos. Esto se debe principalmente, a que las políticas públicas se caracterizan, en la actualidad, por poseer estructuras de intervención mucho más complejas que una binaria. Las evaluaciones que consideran estos tratamientos complejos parecieran acercarse más a la realidad en términos del grado de exposición que reciben las unidades bajo tratamiento.

\section{El Plan Nacer y la Mortalidad Infantil}

\subsection{Definición de la Tasa de Mortalidad Infantil}

La mortalidad infantil comprende las defunciones de personas menores a un año y se divide en mortalidad neonatal y post-neonatal. La primera hace referencia a las defunciones ocurridas en los primeros 27 días de vida, mientras que la segunda va desde el fin del período neonatal hasta el año de vida. Existe una serie de elementos que afectan y determinan el nivel de la misma, entre ellos pueden mencionarse factores biológicos, demográficos, socio-económicos, culturales, ambientales, de atención de la salud y geográficos. La influencia de cada uno de estos

\footnotetext{
${ }^{4}$ El Plan Nacer comienza en el año 2005 en las regiones del noroeste y noreste y a fines del año 2007 se extiende al resto del país. En el año 2010, el plan incorporó la atención integral de Cardiopatías Congénitas; y en mayo del año 2012, las prestaciones de cuidado perinatal y materno neonatal de alta complejidad. Desde agosto del año 2012 el programa se expandió hacia nuevos grupos poblacionales, a saber niños de entre seis y nueve años, adolescentes, y mujeres de entre veinte y sesenta y cuatro años; esta ampliación trae consigo nuevas prestaciones y prácticas que son incorporadas al Nomenclador habitual del Plan Nacer.
} 
factores es disímil en los dos subgrupos mencionados. Mientras que en la mortalidad neonatal prevalecen aquéllos relacionados con las condiciones congénitas, en la mortalidad post-neonatal tienen mayor efecto las condiciones ambientales y socio-económicas. Para este último subgrupo, gran parte de las causas de muerte se deben a problemas vinculados con el medio ambiente y ello es más habitual cuanto más se aleja la muerte del momento del nacimiento (infecciones, diarreas, deshidratación, trastornos respiratorios agudos). ${ }^{5}$

Analizar la mortalidad infantil a lo largo de distintas unidades económicas, como países, provincias, departamentos, localidades, no tiene demasiado sentido, ya que unidades con mayor población tienen una mayor probabilidad de presentar valores más altos de este indicador; lo mismo sucede, si se analiza una misma unidad económica a lo largo del tiempo en el caso de que su población vaya cambiando. Es por eso que se utiliza la tasa de mortalidad, de manera de poder hacer un análisis comparativo entre unidades o en el tiempo. Para el caso de mortalidad infantil, la tasa se define como el cociente entre el número de defunciones y el número de nacidos vivos multiplicado por mil.

Según la Dirección de Estadísticas e Información en Salud (DEIS) las principales causas de mortalidad infantil en Argentina, ordenadas por importancia, son: trastornos relacionados con la duración de la gestación, dificultades respiratorias del recién nacido, otras malformaciones congénitas, ${ }^{6}$ malformaciones congénitas del corazón, presencia de sepsis bacteriana del recién nacido, otras afecciones respiratorias del recién nacido ${ }^{7}$ y resto de las afecciones perinatales ${ }^{8}$. En la Tabla 1 se presentan las principales causas de mortalidad para menores de un año para el año 2004.

Las causas de muerte de los niños menores de un año se agrupan de acuerdo a los criterios de reducibilidad propuestos por el Taller de Grupos de Expertos (Dirección de Maternidad e

\footnotetext{
${ }^{5}$ Para consultar con mayor precisión el significado de algunos de estos términos ver Carrera, Mallafré y Serra (1986) y Sistema de estadísticas socio-demográficas (SESD) de INDEC.

${ }^{6}$ Según la Clasificación Internacional de Enfermedades CIE-10, dentro de este grupo se incluyen las enfermedades congénitas de los ojos, cara y cuello (códigos Q10-Q18), enfermedades congénitas del aparato respiratorio (códigos Q30-Q34), malformaciones de labios, boca y paladar (códigos Q35-Q38), otras malformaciones del tracto digestivo (códigos Q39-Q45), malformaciones de los órganos genitales (códigos Q50-Q56), malformaciones de los órganos urinarios (códigos Q60-Q64) y otras dismorfias congénitas (códigos Q80-Q89) .

${ }^{7}$ Incluye los síndromes de aspiración neonatal, enfisema intersticial y afecciones relacionadas, originadas en el período perinatal, hemorragia pulmonar originada en el período perinatal, enfermedad respiratoria crónica originada en el período perinatal y otros problemas respiratorios del recién nacido, originados en el período perinatal, que corresponden a los códigos P24-P28 de la CIE-10.

${ }^{8}$ Comprende trastornos cardiovasculares originados en el período perinatal (código P29), enfermedades virales congénitas (código P35), otras enfermedades infecciosas y parasitarias congénitas (código P37), otras infecciones específicas del período perinatal (código P39), enfermedades endocrinas transitorias del feto y del recién nacido (códigos P70-P74), enfermedades del sistema digestivo del feto y del recién nacido (códigos P75-P78), enfermedades de la piel y de la regulación de la temperatura (códigos P80-P83), y otras enfermedades del feto y del recién nacido (códigos P90-P96), según la CIE-10.
} 
Infancia, 1996). El concepto de reducibilidad se aplica a las defunciones cuya frecuencia podría disminuirse, en función del conocimiento científico actual y por distintas acciones desarrolladas fundamentalmente a través de los servicios de salud. Estudiar las causas de muerte según criterios de reducibilidad tiene como objeto detectar problemas, sustentar la toma de decisiones y guiar las actividades, de forma tal que faciliten la instrumentación de medidas correctivas adecuadas. En la Argentina esta clasificación comenzó a usarse con datos de 1979 a partir de 1985. En 1996 el agrupamiento de causas de muertes infantiles vigente fue modificado, teniendo en cuenta los avances científicos y tecnológicos y los cambios en las modalidades de atención. En la mortalidad neonatal las defunciones se consideran: a) reducibles por prevención, diagnóstico o tratamiento oportuno (tanto en el embarazo, como en el parto y en el recién nacido), b) otras reducibles, c) difícilmente reducibles y d) otras causas. Por su parte, las defunciones post-neonatales se agrupan en: a) reducibles por prevención, b) reducibles por tratamiento, c) reducibles por prevención y tratamiento, d) otras reducibles, e) difícilmente reducibles, f) desconocidas o mal definidas y g) otras causas.

En las Tablas 2 y 3 se muestra el porcentaje de defunciones agrupadas según criterios de reducibilidad para el año 2004 para la mortalidad neonatal y postneonatal respectivamente. La idea subyacente detrás de este análisis es interpretar correctamente las causas de mortalidad en relación a su potencial reducción, a fin de desarrollar intervenciones eficaces en especial en las acciones que se relacionan con la necesidad de mejorar la calidad y el acceso equitativo a la atención infantil. De ambas tablas se deriva que tanto para la mortalidad neonatal como para los post-neonatal cerca del $57 \%$ de las defunciones son atribuibles a factores reducibles. Estos resultados estarían indicando que existe espacio para el desarrollo de una estrategia de salud orientada a la búsqueda de resultados sanitarios.

\subsection{La Tasa de Mortalidad Infantil en Argentina}

La tasa de mortalidad infantil en Argentina ha mostrado una marcada caída en los últimos diez años. Como se observa en la Figura 1, después de mantenerse en un alto nivel en el período 2000-2002, probablemente debido a la delicada crisis social, económica, e institucional, se produce un quiebre en la tendencia, pasando a ser decreciente, hasta la actualidad. No obstante en el lapso 2005-2007 hubo un cierto estancamiento en esta reducción de la tasa, derivándose varias interpretaciones posibles de este hecho. Una podría ser un colapso de la estructura del sistema de salud, o dicho de otra forma, un exceso de demanda; otra quizás sea que Argentina alcanzó algo similar a una tasa "natural" de mortalidad infantil la cual es muy difícil bajar; sin embargo este 
razonamiento no parecería ser el correcto ya que existen países con una tasa de mortalidad seis veces menor que la de Argentina por ejemplo Singapur, Suecia y Japón.

Cruces et al. (2011) estudian los efectos de las crisis económicas en Argentina sobre una serie de variables de resultados sanitarios. En concreto, encuentran que las crisis empeoran el estado de salud de la población aumentando tanto las tasas de mortalidad infantil y materna como el bajo peso al nacer. En sintonía con esto, Bozzoli et al (2010) muestran que la crisis económica del 2002 lleva a una caída del peso promedio al nacer de 30 gramos, y que la misma afecta en mayor proporción a los niños con madres con un bajo status socioeconómico.

Según datos de la Organización Panamericana de la Salud, Argentina no se encuentra en una mala posición si se rankea a los países americanos según la última tasa de mortalidad infantil reportada (ver Figura 2). Aunque el valor presentado es considerablemente mayor que los reportados por países desarrollados como Canadá y Estados Unidos, es aproximadamente cinco veces menor que el máximo valor alcanzado en la región (Haití). Los números dejan de ser alentadores si se compara Argentina con países de un grado de desarrollo similar como Chile y Uruguay. El país trasandino presenta una tasa de mortalidad infantil que es casi la mitad, mientras que la de Uruguay es tres puntos porcentuales menor que la de Argentina. Brasil, un país al que nunca se puede obviar en la comparación internacional, presenta una tasa de mortalidad sustancialmente mayor que Argentina (seguramente debido a los grandes problemas de pobreza y desigualdad, todavía presentes en ese país), aunque con una gran caída en los últimos quince años. ${ }^{9}$

\subsection{La Mortalidad Infantil a nivel Regional en Argentina}

A nivel regional, la distribución de la mortalidad infantil no presenta un patrón uniforme. Las regiones de NOA y NEA, por ser las que presentan los peores indicadores socioeconómicos, son las que poseen las tasas de mortalidad infantil más elevadas. En la Tabla 4 se presentan una serie de indicadores, entre ellos el porcentaje de nacidos vivos con muy bajo y con bajo peso, el porcentaje de nacidos vivos con madres de 15 y 20 años, la tasa de mortalidad materna y la tasa de mortalidad infantil. En la gran mayoría de los casos las regiones de NOA y NEA poseen los peores resultados.

Más allá de estas diferencias, la composición de la tasa de mortalidad infantil presenta un comportamiento similar en las regiones de NOA y NEA en relación al resto del país. Si se divide la tasa de mortalidad infantil en neonatal y post-neonatal puede observarse que la mayor cantidad de defunciones ocurre en el primer caso. Cerca del 67 por ciento de las defunciones de menores de un año ocurren cuando el bebé posee menos de veintisiete días. Esto sucede tanto en NOA y NEA

\footnotetext{
${ }^{9}$ Ver Naciones Unidas (2010).
} 
como en el resto del país. Estos resultados son importantes, ya que por más que en NOA y NEA las tasas de mortalidad son mayores que en el resto del país, la composición es similar en las distintas regiones. Un análisis detallado abordaría las causas de esas defunciones y su similitud o disparidad entre las diferentes regiones; es por eso que en la Tabla 5 se presentan las principales causas de mortalidad infantil en las distintas regiones que, como puede observarse, no difieren de manera sustancial. En ambos casos, los trastornos relacionados con la duración de la gestación, las dificultades respiratorias del recién nacido, y las malformaciones congénitas, juegan un rol importante en las defunciones de menores de un año. Esto resulta importante sobre todo teniendo en cuenta la estrategia de estimación que se presenta en la siguiente sección. Más allá de que en niveles la tasa de mortalidad infantil entre las regiones NOA y NEA difiere respecto al resto del país, la composición de la misma en las distintas regiones es similar. Esto robustece el rol que juega el grupo de comparación en la estrategia de estimación implementada. Algo similar ocurre con la evolución de las tendencias pre-implementación del programa que se presentan también en la siguiente sección.

\subsection{La Mortalidad Infantil y el Plan Nacer}

Es en el contexto descripto anteriormente, donde se desarrolla el Plan Nacer. Este programa federal, llevado adelante por el Ministerio de Salud de la Nación, invierte recursos para mejorar la cobertura de salud y la calidad de atención de las mujeres embarazadas, puérperas y niños menores de 6 años que no tienen obra social. Se inició en el año 2004 y comenzó a ejecutarse en las provincias del Noroeste y Noreste de la Argentina (NOA y NEA, respectivamente) en 2005, por ser las regiones que presentaban los indicadores socio-sanitarios más desfavorables. Desde fines de 2007, el programa se expandió a todo el país.

Los objetivos del proyecto son, por un lado, incrementar la utilización y la calidad en los servicios de salud para la población a cargo y, por otro lado, mejorar la gestión institucional de los organismos y establecimientos encargados de llevar adelante la política sanitaria. La elección de un conjunto de prácticas priorizadas agrupadas en un Nomenclador y financiadas por el programa, fueron seleccionadas de manera de contribuir al descenso de la mortalidad materna e infantil en la Argentina, aumentando la inclusión social y mejorando la calidad de atención de la población. La novedad del programa radica en la generación de un seguro público de salud para la población materno-infantil sin cobertura social y en la aplicación de un modelo de financiamiento basado en resultados. El sesenta por ciento de los recursos transferidos a las provincias son aportados como producto de la nominalización de la población elegible, lo que implica, básicamente, la identificación e inscripción de los beneficiarios, mientras que el resto depende del grado de 
cumplimiento de una serie de metas sanitarias que se denominan "trazadoras". ${ }^{10}$ Por su parte, las provincias transfieren fondos a los establecimientos en función de la realización de un conjunto de prácticas. Los establecimientos poseen autonomía en el gasto de sus recursos incluso pueden destinar hasta un $50 \%$ en incentivos al personal.

Porto (2006) analiza los flujos de fondos entre los distintos actores del programa. Muestra que las transferencias de la nación a las provincias son condicionadas a un gasto específico, no exigen contrapartida al menos en los primeros tres años, son discrecionales ya que la nación puede modificar unilateralmente los montos y condiciones, y son abiertas ya que el monto total percibido por las provincias no está predeterminado sino que depende de la inscripción de beneficiarios. Según el autor, este escenario "crea fuertes incentivos iniciales para el logro del objetivo en materia de salud". Además plantea que la "implementación del pago por servicios por parte de las provincias a los establecimientos constituye un poderoso incentivo a la productividad".

El Plan Nacer se enmarca dentro de los programas que pagan por resultado. Eichler (2006) entiende a estos programas como transferencias monetarias o materiales en función del cumplimiento de determinadas metas predeterminadas. Meessen, Kashala y Musango (2007) plantean que la pobre respuesta del sistema de salud actual de los países de ingresos medios y bajos, se debe a acuerdos institucionales inadecuados. En ese sentido, argumentan que existe una configuración de contratos que establecen los incentivos que en última instancia determinan la performance del sistema. Dividen a los contratos en aquellos que establecen la forma en que los establecimientos de salud acceden a los recursos físicos "physical resource contracts" y aquellos que establecen el grado de discrecionalidad con que las autoridades de los establecimientos remuneran a sus recursos humanos "governance and employment contracts". Eldridge y Palmer (2008) argumentan que los acuerdos que pagan por resultados están diseñados de manera de que en una relación principal-agente se alineen ambos objetivos. El dilema del principal se resuelve diseñando un contrato que motiva al agente a comportarse en la forma que el principal desea, incluso cuando no puede monitorear de manera plena lo que el agente hace.

Toonen, Canavan, Vergeer y Eloviano (2009) plantean que la asignación de recursos por presupuesto "modality input planning" no incentiva a los proveedores de salud a actuar mejor porque los flujos de dinero no están atados a resultados. La noción del pago por desempeño se

\footnotetext{
${ }^{10}$ Las 10 metas a obtener por cuatrimestre que fueron diseñadas para poder monitorear la evolución y el desarrollo del Proyecto son: (I) Captación temprana de mujeres embarazadas, (II) Efectividad de atención del parto y atención neonatal, (III) Efectividad de cuidado prenatal y prevención de prematurez, (IV) Efectividad de atención prenatal y del parto, (V) Evaluación del proceso de atención de los casos de muertes, (VI) Cobertura de inmunizaciones, (VII) Cuidado sexual y reproductivo, (VIII) Seguimiento de niño sano hasta 1 año, (IX) Seguimiento de niño sano de 1 a 6 años y (X) Inclusión de la población indígena.
} 
sustenta en la creencia de que los agentes están motivados por ganancias financieras y buscan formas de maximizar sus ingresos.

El Plan Nacer es uno de los primeros programas de pago por resultado con un alcance de escala nacional. En la gran mayoría de los casos, este tipo de programas han sido pruebas piloto sin una escala considerable. Uno de los pocos países que ha pasado de una intervención piloto a una ampliación de alcance nacional es Ruanda. No existen evaluaciones metodológicamente robustas de este tipo de intervenciones; incluso, los oponentes a los programas que pagan por resultado plantean que éstos no se sustentan en evidencia rigurosa. El trabajo de Basinga, Gertler, Binagwaho, Soucat, Sturdy y Vermeersch (2010) es una de las pocas referencias existentes de evaluaciones de programas que pagan por resultado.

\section{Estimación y Resultados}

\subsection{Estrategia de estimación}

El Plan Nacer es un programa público y, como tal, debe estar fundamentado en una teoría de cambio que relacione la intervención con los resultados de interés, en línea con lo expuesto por Imas y Ray (2009). Mientras que la teoría de cambio es una descripción de cómo se supone que una intervención conseguirá los resultados, la evaluación de impacto mide si esos resultados fueron logrados. La teoría de cambio subyacente es que el Plan Nacer genera, a través de una configuración de convenios y contratos, una correcta asignación de incentivos. El pago por resultados resuelve los problemas de agencia existentes entre los distintos actores involucrados en el programa, generando una mejora en el acceso a los servicios de salud y en la calidad de las prestaciones. Consecuentemente es de esperarse que la población a cargo mejore su condición sanitaria.

El trabajo sigue un enfoque no-experimental para evaluar los efectos del Plan Nacer sobre la mortalidad infantil. Se propone seguir una metodología de diferencias en diferencias que consiste básicamente en la comparación de condiciones sanitarias entre poblaciones inscriptas y no inscriptas al programa. La fuente principal de variación es la participación en el programa a nivel departamental. ${ }^{11}$ Esta variación a nivel departamental existe solamente por un plazo limitado, en particular, entre el comienzo del programa en las provincias del noroeste y del noreste argentino en el año 2005 hasta la ampliación al resto de las provincias a fines del año 2007. El momento en que debe evaluarse un programa depende, en gran medida, del tipo de intervención a ser evaluada. Probablemente una programa que busque mejorar la asistencia escolar, requiera de una evaluación

\footnotetext{
${ }^{11}$ En total en Argentina existen 511 departamentos distribuidos a lo largo de las distintas provincias. Para las regresiones también se consideran las 28 circunscripciones electorales en que se divide la Ciudad Autónoma de Buenos Aires.
} 
al poco de tiempo de haberse iniciado el mismo. Por el contrario, si el objetivo es mejorar el acceso al mercado laboral, seguramente se necesite una evaluación a más largo plazo. En ese sentido es crucial evaluar las variables de resultado a ser analizadas para tomar una decisión acertada. En este estudio, el problema se resuelve de forma metodológica. Dado que a fines de 2007 el programa se expande a todo el país, la imposibilidad de contar con un grupo de comparación válido, inhabilita la utilización de la metodología presentada, y por ende hace imposible la evaluación. Más allá de que a priori tres años de tratamiento parezca un período algo acotado, se espera un efecto, sobre todo teniendo en cuenta el tipo de prestaciones que son financiadas. ${ }^{12}$

Para aplicar una metodología de diferencias en diferencias se necesita, en el caso más simple, información para ambos grupos (tratamiento y control) al menos para dos momentos en el tiempo (previo a la intervención y después de la misma). En el caso particular de este trabajo, el grupo de tratamiento está conformado por aquellos departamentos que pertenecen a provincias del NOA-NEA y el grupo de control por el resto de los departamentos de la Argentina. En relación a la estructura temporal de los datos con que se trabaja, se cuenta con información previa a la implementación del Plan Nacer, más específicamente para el año 2004, y con información posterior a la misma (año 2007). ${ }^{13}$ El supuesto de identificación es que en ausencia del tratamiento las tendencias para ambos grupos fueron similares. Si ambas tendencias se comportaron de manera similar antes de la implementación del Plan Nacer, esto quiere decir que la diferencia en niveles se mantuvo relativamente estable a lo largo de un determinado período de tiempo. Si, además, no hubo factores más allá del programa que afectaron las tendencias de los dos grupos por separado, entonces si existió una diferencia en niveles distinta a la situación pre-programa, probablemente se debió a la implementación del mismo.

En la Figura 3 se presenta la evolución de la tasa de mortalidad infantil para los dos grupos (léase NOA y NEA, versus el resto). Aunque las dos series son sustancialmente distintas en niveles, su tasa de cambio es similar y ambas parecen moverse a la par. Esto estaría indicando que si la diferencia en niveles cambia después del plan en relación a como se venía comportando antes

\footnotetext{
${ }^{12}$ Tal cual se deriva del Manual Operativo (Ministerio de Salud de la Nación, 2004), el conjunto de prestaciones que fueron incluidas en el Nomenclador Único, son consideradas de alto impacto y costo efectividad en la salud de la madre y el niño. Algunas de las prestaciones incluidas en el nomenclador son: consulta de control prenatal de primera vez, consulta ulterior de control prenatal, atención de parto y recién nacido, cesárea y atención del recién nacido, consulta de puerperio inmediato, consulta pediátrica menores de un año, entre otras.

${ }^{13}$ Notar que el grupo de tratamiento, conformado por aquellos departamentos que pertenecen al resto del país, a fines de 2007, ya se encontraba bajo programa. Sin embargo, se supone que esto no altera las defunciones correspondientes al mismo año, debido a que solo se habían incorporado muy pocos establecimientos y durante un lapso de tiempo reducido. King y Behrman (2008), consideran que las intervenciones no son instantáneas, sobre todo porque estas invocan cambios de comportamientos en los proveedores de servicios y en sus usuarios.
} 
de su introducción, en ausencia de algún factor que afecte a alguna de las dos sub-muestras en particular, el plan lleva a una reducción de la tasa de mortalidad infantil de las regiones del Noroeste y Noreste argentino en relación al resto del país. Este gráfico le otorga robustez al supuesto de identificación presentado en el párrafo anterior. Más específicamente, si las tendencias pre implementación del plan hubiesen sido totalmente distintas, la aplicación de diferencias en diferencias como metodología de estimación no hubiese sido la correcta.

El tratamiento utilizado considera distintos grados de exposición de las unidades bajo programa. Como fue mencionado anteriormente, la literatura de evaluación de programas sociales ha mutado desde un enfoque de tratamiento binario a uno que considera distintos grados de exposición. Básicamente esto se debe a que las políticas públicas se caracterizan, en la actualidad, por poseer estructuras de intervención mucho más complejas que una binaria. Las evaluaciones que consideran estos tratamientos complejos parecieran acercarse más a la realidad en términos del grado de exposición que reciben las unidades bajo tratamiento. En ese sentido, la razón de utilizar este enfoque para el estudio se funda en el hecho de que en realidad no todos los departamentos se ven afectados de igual forma. El Plan Nacer se materializa a través de los establecimientos de salud que brindan las prestaciones presentes en el Nomenclador Único, y la distribución de estos a lo largo de los departamentos dista de ser homogénea invalidando de esta forma la utilización de un tratamiento binario.

La información con la que se cuenta proviene de dos fuentes. Por un lado, la información de defunciones, población y mortalidad que proviene de los Informes de Estadísticas Vitales elaborado por DEIS (Dirección de Estadísticas e Información en Salud). En dichos informes se presenta información estadística sobre nacidos vivos, defunciones totales, por grupos de edades y defunciones maternas, por lugar de residencia habitual, agrupados según departamento. Por otro lado, se utiliza información de gestión y monitoreo del programa del Ministerio de Salud de la Nación que contiene los establecimientos con convenio con el programa. Esta información a nivel establecimiento permite caracterizar a los mismos en función de distintos aspectos, entre ellos, el tipo de establecimiento, su dependencia administrativa y si son rurales o urbanos. ${ }^{14}$

La utilización de datos administrativos posee ciertas ventajas por sobre la utilización de datos recolectados a través de encuestas. En primer lugar y sobre todo en aquellos estudios que utilizan información referida a dos momentos distintos en el tiempo, no existen problemas vinculados con atrición (attrition), que se refiere a la imposibilidad de recolectar información de una unidad económica en algún relevamiento posterior a la línea de base. Este tipo de problema

\footnotetext{
${ }^{14}$ El tipo de establecimiento se refiere a la clasificación utilizada por el Plan Nacer para agrupar a los establecimientos de salud.
} 
conjuntamente con al abandono de los participantes durante el tratamiento puede resultar en estimaciones sesgadas. Una segunda ventaja, no menor, se vincula con el hecho de que la utilización de datos administrativos es sustancialmente más barata que la que proviene de encuestas. La principal desventaja se relaciona con el hecho de que son recolectados por agencias del propio programa o agencias gubernamentales que llevan adelante el mismo. Galasso y Yau (2006) enfatizan que cada vez con mayor frecuencia es utilizada esta fuente de información sobre todo en los programas de asistencia pública en los países en vías de desarrollo. Además, destacan la utilización de información de monitoreo del programa, definida como un subconjunto de información administrativa dirigida a actividades específicas del programa, para evaluar el impacto de un programa.

Más allá de lo argumentado en relación a la utilización de distintos grados de exposición, se analiza en una primera instancia, el impacto del Plan Nacer considerando un tratamiento binario. Este tratamiento es de fácil aplicación e interpretación ya que trata a todas las unidades bajo programa de la misma forma, suponiendo que el grado de exposición al programa, en magnitud o extensión por ejemplo, es el mismo para todas las unidades tratadas.

El modelo propuesto es entonces el que se presenta en la siguiente ecuación:

$y_{t i}=\alpha+\beta d_{i} * t_{t}+\delta x_{t i}+\phi_{i}+\varsigma_{t}+\mu_{t i}$

donde la variable dependiente es la tasa de mortalidad infantil, $d$ es una variable binaria que refleja la condición frente al tratamiento (igual a uno para las unidades tratadas), las $x$ se corresponden con la población y la cantidad de nacidos vivos, mientras que $\phi$ se corresponde con un efecto fijo y $\varsigma$ con un efecto temporal. El subíndice $i$ indexa a los departamentos, la unidad de análisis en el presente trabajo, mientras que el subíndice $t$ se refiere al período temporal en cuestión. ${ }^{15}$

La metodología de diferencias en diferencias implicaría realizar una regresión de la siguiente ecuación, teniendo en cuenta el modelo anterior:

$$
\Delta y_{i}=y_{t i}-y_{t-1 i}=\left(\zeta_{t}-\zeta_{t-1}\right)+\beta d_{i}\left(t_{t}-t_{t-1}\right)+\delta\left(x_{t i}-x_{t-1 i}\right)+\mu_{t i}-\mu_{t-1 i}
$$

lo que elimina cualquier factor correlacionado con la asignación del tratamiento que se mantiene constante a lo largo de los dos períodos seleccionados. Junto con los controles mencionados, se agrega la intensidad inicial de los establecimientos, entendida ésta como el cociente entre los establecimientos de salud públicos y la población de cada departamento, y la tasa de mortalidad infantil del período base. ${ }^{16}$

\footnotetext{
${ }^{15}$ La población esta expresada en miles.

${ }^{16}$ Se incluyen dentro de los establecimientos públicos de salud a todos aquellos en condiciones de brindar las prestaciones presentes en el nomenclador financiado por el programa. Prácticamente la totalidad de los establecimientos públicos de salud están bajo esa condición.
} 
En la tabla 6 se presentan los resultados de estas regresiones. Como se observa, el programa tiene un efecto cuando se controla tanto por el cambio poblacional entre los dos años seleccionados como por el cambio en la cantidad de nacidos vivos. Ese efecto se mantiene incluso controlando por la intensidad inicial de los establecimientos de salud; este control involucra a todos los establecimientos de salud públicos más allá de si están bajo programa o no, y se define como el cociente entre los establecimientos y la población relativa a cada departamento. Sin embargo, si se controla por la tasa de mortalidad inicial, de manera de controlar por diferencias en la tendencia que son una función de la tasa de mortalidad, el efecto del programa deja de ser significativo.

Como bien destacan King y Behrman (2008), la gran mayoría de las evaluaciones tratan a las intervenciones como si fuesen instantáneas, con cambios predecibles e iguales a lo largo de todos los grupos tratados. Muchas evaluaciones asumen implícitamente que el impacto sobre los individuos es dicotómico, esto es que los individuos están expuestos o no. Sin embargo, cuando el tratamiento involucra una operatoria complicada que requiere una organización compleja, el supuesto incorrecto es que el tratamiento ocurre en una determinada fecha y que es implementado completamente y de la misma forma a lo largo de todos los tratados. En el caso del Plan Nacer, la cantidad de establecimientos relativo a su población difiere sustancialmente entre departamentos lo que vuelve insostenible el supuesto de un tratamiento binario.

Se realiza entonces el análisis incorporando distintos grados de exposición al programa. El modelo es igual al anterior salvo que $d$, en este caso, es una variable que refleja el grado de exposición del departamento frente al programa. En ese sentido esta variable se genera como el cociente entre la cantidad de establecimientos con convenio a diciembre de 2007 y la población del departamento. Se le suma a los controles del caso binario la intensidad inicial de los establecimientos públicos de salud, la tasa de mortalidad infantil inicial, y un control que identifica los departamentos que pertenecen a las regiones bajo tratamiento.

El punto central pasa entonces por demostrar que el grado de exposición es exógeno. Esto es así por varias causas. En primer lugar los establecimientos con convenio, o los que primero entran al programa, son los más grandes o los que atienden a más personas. Esto se sustenta en el hecho de que las provincias buscan maximizar sus ingresos, por lo tanto en una primera instancia lo lógico es suponer que primero entren los que potencialmente generen mayores ingresos. Entonces, si se controla por la población, se sostiene la exogeneidad del tratamiento. En segunda instancia, los departamentos carecen de autoridades ejecutivas que puedan generan alguna influencia sobre los efectores con convenio, esto eliminaría la potencial correlación entre los efectores con convenio y la tasa de mortalidad infantil. Tercero, el ministro de salud nacional fue el mismo para todo el período bajo análisis (2004-2007) y ni los gobernadores provinciales ni los ministros de salud provinciales 
cambiaron, por lo que tampoco existe correlación por este aspecto. ${ }^{17}$ Por último, las características propias de cada departamento que se correlacionan con el tratamiento, y que se mantienen constantes entre ambos períodos, son eliminadas con los efectos fijos.

En la Tabla 7 se presentan los resultados inherentes a estas regresiones. Los factores biológicos, culturales, ambientales y geográficos que afectan la mortalidad se controlan por los efectos fijos, los factores demográficos son capturados por el cambio poblacional y de nacidos vivos, y los factores de atención de la salud por la intensidad inicial de los establecimientos públicos de salud. Todos los mecanismos que sesgan la regresión como por ejemplo el porcentaje de personas con necesidades básicas insatisfechas y otros factores socioeconómicos, están capturados por el nivel inicial de mortalidad.

Un mayor grado de exposición al programa, representado por el cociente entre los establecimientos con convenio y la población, conlleva a una caída en la tasa de mortalidad infantil. El coeficiente resulta negativo y significativo incluso después de realizar una serie de controles. El cambio poblacional es uno de los controles de mayor importancia, ya que como se argumentó anteriormente permite que la participación en el programa de los establecimientos de salud sea exógena. La tasa de mortalidad infantil del período inicial y la intensidad de los establecimientos públicos de salud explican gran parte del cambio en la variable de interés. La primera soslaya el comportamiento autorregresivo que tiene este tipo de indicadores y controla por los factores socioeconómicos que pueden estar sesgando la regresión, mientras que la segunda resalta el hecho de que la política sanitaria, materializada en la disponibilidad de centros de atención primaria, es crucial en la disminución de la tasa de mortalidad infantil.

\subsection{Experimentos de falsificación}

La validez de los supuestos utilizados en la estrategia de estimación puede comprobarse, al menos parcialmente, en evaluación de impacto. Esto se logra con experimentos de falsificación que permiten mejorar la confianza en los supuestos. Mientras que no superar estas pruebas implica que los supuestos no fueron utilizados de manera adecuada, hacerlo confirma parcialmente los mismos, ya que nunca se puede estar seguro de su validez.

Se propone, entonces, explorar varios ejercicios de validación. En la Tabla 8 se presentan las mismas regresiones que en la Tabla 7 pero para los años 2001 y 2004. Este experimento consiste

\footnotetext{
${ }^{17}$ Todas las provincias tienen el mismo gobernador y vice gobernador entre 2003 y 2007, salvo las provincias de Corrientes y Santiago del Estero. En Corrientes cambian las autoridades, pero se mantiene el mismo partido político en el poder. Fuente: Ministerio del Interior de la Nación. República Argentina. Esta información se encuentra disponible en http://www.mininterior.gov.ar/provincias/provincias.php?idName=provincias\&idNameSubMenu=\&idNameS $\underline{\text { ubMenuDer }}=$
} 
en realizar una estimación utilizando un tratamiento falso, es decir, para un grupo que no fue afectado por el programa: se asigna un tratamiento a las mismas unidades económicas pero en un período falso. Si el supuesto de identificación es válido entonces no debería haber una relación entre el tratamiento y la tasa de mortalidad infantil para los dos años seleccionados ya que el programa no estaba en ejecución. Efectivamente, los datos muestran que no existe una relación estadística entre el tratamiento y la tasa de mortalidad infantil.

Otro ejercicio de validación, quizá el más utilizado a los fines de este enfoque, surge de analizar las tendencias pre-intervención y comparar si las mismas presentan un comportamiento similar. Esto se hizo en la sub-sección 4.1, comprobando que ambas tendencias presentan un comportamiento pre-programa similar.

\subsection{Exposición de los departamentos vecinos}

Una posibilidad es que en las regresiones presentadas en la sección anterior, el grado de exposición de un departamento en particular no capture el alcance total del programa ya que los individuos pueden concurrir a establecimientos de salud de departamentos vecinos; resultaría lógico que las familias se trasladen en búsqueda de una mejor cobertura de salud a departamentos que se encuentran geográficamente cerca.

El hecho de que las familias tengan la posibilidad de desplazarse a departamentos vecinos para encontrar respuestas a sus problemas de salud, motiva a incorporar en las regresiones anteriores no sólo el grado de exposición de cada departamento sino que también debería incorporarse el grado de exposición de los departamentos vecinos, de manera de controlar por un potencial movimiento de individuos entre departamentos en búsqueda de mejores condiciones de atención sanitaria. Para construir esta variable es necesario localizar geográficamente a cada uno de los departamentos de la base y construir una matriz de ponderadores, que se definen en función de cuán cerca o lejos está un departamento de otro. La información referida a la localización de cada uno de los departamentos es obtenida de DIVA-GIS (Geographic Information System por sus siglas en inglés). ${ }^{18}$ En concreto, lo que se obtiene de DIVA-GIS son los límites administrativos de cada uno de los departamentos del país. A partir de esta información se calcula el centroide de cada una

18 DIVA-GIS es apoyada por diversas instituciones del CGIAR (Consultative Group on International Agricultural Research) entre ellas Bioversity International, el International Potato Center, el International Rice Research Institute, por la Universidad de California, Berckeley, y por el Museum of Vertebrate Zoology. Se puede acceder de manera gratuita a la información en el siguiente link http://www.diva-gis.org/ 
de los departamentos de manera de tomar un punto de referencia para calcular la cercanía o lejanía entre ellos. ${ }^{19}$

Con esta información se calcula la matriz de ponderadores espaciales. En primer lugar se calcula la distancia euclidiana de cada uno de los centroides con respecto al resto. ${ }^{20}$ En segundo lugar se asigna un factor de ponderación en función de la distancia, así un centroide que se encuentra "cerca" de otro va a tener una alta ponderación. Sólo se consideran los 5 departamentos que poseen la menor distancia. Esta elección permite que las personas se desplacen en búsqueda de una mejor atención sanitaria pero elimina el caso en que un individuo se traslade grandes distancias. La ponderación de dos departamentos que están contiguos es alta en relación a dos ubicados más lejos. En la Tabla 10 se presentan los resultados incorporando estas consideraciones. Específicamente, en este nuevo escenario, se considera el grado de exposición al programa de un departamento en particular como la suma de su propio grado de exposición y la suma ponderada del grado de exposición de los 5 departamentos más cercanos. ${ }^{21}$ En este nuevo escenario el efecto del programa sigue siendo negativo y significativo.

\subsection{Mortalidad Neonatal y Mortalidad Post-neonatal}

En este apartado se analiza la presencia de un impacto diferencial del Plan Nacer sobre la tasa de mortalidad neonatal (aquellas defunciones ocurridas dentro de los primeros veintisiete días) y la mortalidad post neonatal (aquellas ocurridas entre los veintisiete días y el año de vida). Como se planteó en la sección 3 existen distintos factores que influyen y determinan el nivel de las mismas. Mientras que en la mortalidad neonatal prevalecen aquéllos relacionados con las condiciones congénitas, es decir las condiciones que el recién nacido adquiere durante su vida intrauterina, en la mortalidad post-neonatal tienen mayor efecto las condiciones ambientales y socio-económicas sobre la salud del niño. Para este último subgrupo, gran parte de las causas de muerte se deben a problemas vinculados con el medio ambiente y ello es más habitual cuanto más

\footnotetext{
${ }^{19}$ Un centroide de un polígono de $n$ vértices definido como $\left(x_{0}, y_{0}\right),\left(x_{1}, y_{1}\right), \ldots,\left(x_{n-1}, y_{n-1}\right)$ es el punto $\left(C_{\mathrm{x}}, C_{\mathrm{y}}\right)$, tal que $C_{x}=\frac{1}{6 A} \sum_{i=o}^{n-1}\left(x_{i}+x_{i+1}\right)\left(x_{i} y_{i+1}-x_{i+1} y_{i}\right)$ $C_{y}=\frac{1}{6 A} \sum_{i=o}^{n-1}\left(y_{i}+y_{i+1}\right)\left(x_{i} y_{i+1}-x_{i+1} y_{i}\right)$

Donde A es el área del polígono $A=\frac{1}{2} \sum_{1=0}^{n-1}\left(x_{i} y_{i+1}-x_{i+1} y_{i}\right)$

${ }^{20}$ Para un espacio bidimensional, la distancia euclidiana entre dos puntos $\mathrm{P}_{1}$ y $\mathrm{P}_{2}$ con coordenadas $\left(\mathrm{x}_{1}, \mathrm{y}_{1}\right)$ y $\left(\mathrm{x}_{2}, \mathrm{y}_{2}\right)$ respectivamente está dada por $d_{E}\left(P_{1}, P_{2}\right)=\sqrt{\left(x_{2}-x_{1}\right)^{2}\left(y_{2}-y_{1}\right)^{2}}$

${ }^{21}$ Como punto de referencia para evaluar cuán lejos o cerca está un departamento de otro se considera el centro de cada departamento.
} 
se aleja la muerte del momento del nacimiento (infecciones, diarreas, deshidratación, trastornos respiratorios agudo).

En la Tabla 11 se presentan las regresiones considerando como variable dependiente la tasa de mortalidad neonatal; como se observa, el programa no tiene un efecto sobre la misma. Probablemente esto se deba a que el Plan Nacer no ha podido atacar las causas más duras de la mortalidad infantil que se encuentran en el período neonatal entre las que resaltan las cardiopatías congénitas. Las causas que prevalecen sobre esta mortalidad se caracterizan por su difícil prevención y tratamiento, por lo que requieren de una atención integral del problema no sólo de mayor prevención sino también de intervenciones eficaces y a tiempo. ${ }^{22}$ El Plan Nacer, a través de sus prestaciones financiadas, tiene un enfoque orientado a la prevención careciendo, de esta forma, de la integralidad en la atención necesaria en este tipo de mortalidad. Consciente de esto, el programa entra en el año 2010 en un proceso de ampliación en el eje de prestaciones, incorporando la cobertura de cardiopatías congénitas. En la misma línea, en mayo del año 2012 se incorporaron prestaciones de cuidado perinatal y materno de alta complejidad. Un próximo estudio deberá evaluar si esta incorporación de nuevas prestaciones genera algún efecto sobre la mortalidad neonatal.

Por otro lado, en la Tabla 12, se presentan las regresiones utilizando la tasa de mortalidad post neonatal como variable dependiente; aquí sí el programa tiene un impacto negativo y significativo. Este tipo de defunciones que pueden ocurrir por enfermedades infecciosas o diarreas, entre otras, puede reducirse, a diferencia de las neonatales, de manera relativamente rápida con intervenciones adecuadas. La disponibilidad de recursos en manos de los establecimientos gracias al programa permite mejorar en gran medida las condiciones bajo las cuales brindan sus prestaciones. Esto es sumamente importante, ya que como se mencionó, en la mortalidad post neonatal prevalecen las condiciones ambientales y socio económicas donde el recién nacido desarrolla sus primeros pasos. No sólo generan mejores condiciones para los usuarios de los servicios sino que también genera incentivos en los prestadores de salud (enfermeras, pediatras, médicos, entre otros) ya que hasta el $50 \%$ de los fondos generados por el programa pueden distribuirse como incentivos al personal.

En las Tablas 13 y 14 se presentan los experimentos de falsificación para las tasas de mortalidad neonatal y post neonatal utilizando una intervención falsa. Nuevamente, los resultados son los esperados y el programa no tiene efecto bajo una intervención falsa.

\footnotetext{
${ }^{22}$ Ver Andrada, Bertone y Peranovich (2008).
} 


\subsection{Mortalidad materna}

La mortalidad materna, definida como la defunción de una mujer mientras está embarazada o dentro de los 42 días siguientes a la terminación del embarazo, es uno de los problemas de investigación en salud más importantes, no sólo en lo referido a la determinación de sus causas sino también a los distintos factores que influyen sobre ella. Calderón, Bustamante, Campuzano y Camarena (2007) quienes realizan un estudio sobre la mortalidad materna a partir del análisis de los expedientes clínicos y de las autopsias verbales, sostienen que la evidencia disponible en la actualidad justifica las muertes maternas como el resultado de un conjunto de problemas sociales, económicos, biológicos y logísticos de los servicios de salud. Argumentan que, en la gran mayoría de los casos, estas muertes ocurren por un indebido acceso a los beneficios de la medicina "moderna" o por una aplicación defectuosa de los conocimientos y tecnologías disponibles en el manejo del embarazo, del parto o del puerperio.

Más allá de que hasta el momento el análisis se centró en el Plan Nacer y su impacto sobre la salud del niño, es interesante ver qué sucede con la mortalidad materna que también forma parte de la población objetivo del programa. La tasa de mortalidad materna refleja el riesgo de morir de las mujeres durante la gestación, el parto y el puerperio. Se define como la cantidad de mujeres fallecidas sobre el número de nacidos vivos como una aproximación al número de mujeres con probabilidad de morir por causas relacionadas con el embarazo, el parto y/o el puerperio. Según DEIS las muertes maternas pueden subdividirse en dos subgrupos:

- Defunciones obstétricas directas: son las que resultan de complicaciones obstétricas del estado de gestación (embarazo, trabajo de parto y puerperio), de intervenciones, de omisiones, de tratamiento incorrecto, o de una cadena de acontecimientos originada en cualquiera de las circunstancias mencionadas.

- Defunciones obstétricas indirectas: son las que resultan de una enfermedad existente desde antes del embarazo o de una enfermedad que evoluciona durante el mismo, no debidas a causas obstétricas directas pero sí agravadas por los efectos fisiológicos del embarazo.

En la Tabla 15 se presentan los resultados considerando la mortalidad materna como variable dependiente. Como queda en evidencia, el Plan Nacer no tiene un efecto sobre la misma. Sin lugar a dudas, esta es una faceta en la cual el programa necesita dirigir sus esfuerzos. De todas formas Argentina no parece ser la excepción; según el "Tracking progress in maternal, newborn and child survival" elaborado por UNICEF (2008), los progresos en materia de disminución de la mencionada tasa han sido marginales en el los últimos años. Según DEIS, más del $30 \%$ de las defunciones maternas en Argentina ocurren por abortos. En principio, el Plan Nacer no debería afectar la tasa de abortos ya que dentro de las prestaciones priorizadas no existe ninguna que 
permita abordar esta problemática. Lo adecuado en este caso, sería una intensiva campaña en salud sexual y reproductiva. De todas formas el programa debería generar una reducción de las muertes por hemorragias o infecciones en el momento del parto o en el puerperio cosa que no ocurre.

\section{Conclusiones}

El Plan Nacer es un programa que invierte recursos en salud con el objetivo de mejorar la cobertura y calidad de los servicios de salud en los niños menores a seis años, las mujeres embarazadas y puérperas que no poseen obra social. Este programa forma parte de una estrategia del ministerio de Salud de la Nación Argentina; se inició en el año 2005 en las provincias del noroeste y noreste del país argentino, para luego a fines de 2007, extenderse a todo el territorio nacional.

La novedad del mismo radica en la generación de un seguro público de salud para la población materno-infantil sin cobertura social y en la aplicación de un modelo de financiamiento basado en resultados. En él intervienen múltiples actores entre los que se destacan la nación, las provincias y los establecimientos de salud. Existen dos tipos de relaciones que determinan los flujos de fondos en la estrategia de financiamiento implementada. La primera relación está dada entre el gobierno nacional y las provincias; el sesenta por ciento de los recursos transferidos a las provincias son aportados como producto de la nominalización de la población elegible para el programa, lo que implica, básicamente, la identificación e inscripción de los beneficiarios, mientras que el resto depende del grado de cumplimiento de una serie de metas sanitarias que se denominan "trazadoras". Por otro lado, los seguros provinciales, que tienen a su cargo la implementación del Plan Nacer en sus territorios, transfieren fondos a los establecimientos de salud en función de un conjunto de prácticas priorizadas que brindan a su población a cargo, agrupadas en el Nomenclador Único.

Este trabajo investiga los efectos del Plan Nacer sobre la mortalidad infantil en la Argentina siguiendo un enfoque no-experimental, basándose particularmente en la metodología de diferencias en diferencias, que consiste en la comparación de condiciones sanitarias entre poblaciones inscriptas y no inscriptas al programa. La fuente principal de variación es la participación en el programa a nivel departamental. Esta variación a nivel departamental existe solamente por un plazo limitado, en particular, entre el comienzo del programa en las provincias del noroeste y del noreste argentino en el 2005 hasta la ampliación al resto de las provincias a fines del 2007.

El tratamiento utilizado considera distintos grados de exposición de las unidades bajo programa. Dado que las políticas públicas se caracterizan en la actualidad, por poseer estructuras de intervención mucho más complejas que una binaria, las evaluaciones que consideran estos 
tratamientos complejos, parecieran acercarse más a la realidad en términos del grado de exposición que reciben las unidades bajo tratamiento. En ese sentido la razón de utilizar este enfoque para el estudio, se funda en el hecho de que en realidad no todos los departamentos se ven afectados de igual forma por el programa. El Plan Nacer se materializa a través de los establecimientos de salud que brindan las prestaciones presentes en el Nomenclador Único, y la distribución de estos a lo largo de los departamentos dista de ser homogénea invalidando de esta forma la utilización de un tratamiento binario.

Los resultados encontrados indican que el Plan Nacer tiene un efecto en la reducción de la tasa de mortalidad infantil. Más específicamente, el Plan mejora el acceso a los servicios de salud y la calidad de las prestaciones, reduciendo la tasa de mortalidad de los niños menores de un año. El resultado es ambiguo si se divide a la tasa de mortalidad infantil entre la tasa de mortalidad neonatal y la tasa de mortalidad post-neonatal. Mientras que el Plan tiene un impacto negativo sobre la segunda, no ocurre lo mismo con la primera. Además, el efecto del programa sobre la tasa de mortalidad materna, no resulta estadísticamente significativo por lo que no existe impacto sobre este indicador.

Existen una serie de aspectos interesantes vinculados con el Plan Nacer que merecen ser evaluados en trabajos futuros. Uno de ellos es analizar cómo impacta el programa sobre la morbilidad materno infantil, es decir sobre la carga de enfermedades (probablemente esto resulte algo complejo sobre todo en términos de disponibilidad de información). Un segundo punto se relaciona con evaluar si el programa genera algún tipo de externalidades o bien, sobre los grupos que no forman parte de la población beneficiaria, o sobre los establecimientos de salud en el sentido de que mejoran su proceso de facturación y esto les permite obtener mayores recursos (por ejemplo explotando con convenios con obras sociales). Otro punto interesante vinculado puntualmente con la generación de mejoras en el diseño del programa, es evaluar bajo qué circunstancias el programa genera mejores resultados (por ejemplo para departamentos con determinadas características). Un último punto es evaluar las distintas ampliaciones que ha tenido el programa. Desde la ampliación al resto del país, a la incorporación de prestaciones orientadas a cardiopatías congénitas y a la atención materno infantil de alta complejidad, hasta la ampliación a nuevos grupos poblacionales. 


\section{Referencias}

Alderman, H. y J. Behrman (2004). "Estimated economic benefits of reducing low birth weight in low income countries" HNP Discussion Paper 35548, World Bank.

Alzúa, M. L., H. Djebbari y M. Valdivia (2012). "Impact Evaluation for Policy Making: A Close Look at Latin American Countries with Weaker Research Capacities". Documento de trabajo $\mathrm{N}^{\mathrm{o}} 132$, CEDLAS.

Andrada M., C. Bertone y A. Peranovich (2008). "Mortalidad infantil, causas y determinantes. Una perspectiva comparada entre la ciudad de Córdoba (Argentina) y Campiñas (Brasil), para el período 2000-2005”. III Congreso de la Asociación Latinoamericana de Población, ALAP, Córdoba, Argentina, del 24 al 26 de Septiembre de 2008.

Basinga, P., P. Gertler, A. Binagwaho, A. Soucat, J. Sturdy y C. Vermeersch (2010) "Paying Primary Health Care Centers for Performance in Rwanda" Policy Research Working Paper 5190, World Bank

Bouillon, C. P y L. Tejerina (2007). "Do we Know what works? A Systematic Review of Impact Evaluations of Social Programs in Latin America and the Caribbean". Inter-American Development Bank Publications $N^{\circ} 23598$.

Bozzoli C. y C. Quintana-Domeque (2010) "The Weight of the Crises: Evidence from Newborns in Argentina” IZA Discussion Paper No. 5294

Calderón, M. A., P. Bustamante, M. Campuzano y A. Camarena (2007) “Aspectos sociales de la mortalidad materna. Estudio de caso en el Estado de México”. Medicina Social, Vol. 2(4).

Carrera, J. M., J. Mallafré y B. Serra (1986). Protocolos de Obstetricia y Medicina Perinatal del Instituto Universitario Dexeus. ELSEVIER-MASSON.

Cruces, G, P. Glüzmann y L. López Calva (2011) "Economic Crises, Maternal and Infant Mortality, Low Birth Weight and Enrollment Rates: Evidence from Argentina's Downturns". World Development Vol. 40 No.2, pp. 303-314 2012.

Dirección de Estadísticas de Salud del Ministerio de Salud de la Nación (2000-2009). Defunciones de menores de cinco años indicadores seleccionados. Argentina. Disponible en http://www.deis.gov.ar/ 
Dirección de Estadísticas de Salud del Ministerio de Salud de la Nación (2000-2009). Estadísticas Vitales. Argentina. Disponible en http://www.deis.gov.ar/

Dirección de Estadísticas de Salud del Ministerio de Salud de la Nación (2000-2009). Indicadores de Salud Seleccionados. Argentina. Disponible en http://www.deis.gov.ar/

Dirección de Maternidad e Infancia (1996). "Taller Grupo de Expertos para la revisión de la clasificación usada en la Argentina sobre Mortalidad Infantil según Criterios de Evitabilidad." Ministerio de Salud y Acción Social, Dirección de Estadísticas de Salud, Comisión Nacional de Clasificación de Enfermedades, Serie 3, Nro.30. Buenos Aires, Argentina.

Eichler, R. (2006). "Can "Pay-for-Performance", increase utilization by the poor and improve the quality of health services?" Discussion paper for the first meeting of the Working Group on Performance-Based Incentives.

Eldridge, C. y N. Palmer (2008). "Performance-based payment: some reflections on the discourse, evidence and unanswered questions". Health Policy and Planning, Vol. 24:160-166.

Evaluation Gap Working Group (2006). "When will we ever learn? Improving lives through Impact Evaluation”. Report of the Evaluation Gap Working Group. Center for Global Development.

Feinstein, O. (2007). “Evaluación Pragmática de Políticas Públicas”. Evaluación de Políticas Públicas Nº36, Información Comercial Española (ICE).

Galasso, E. y J. Yau (2006). "Learning through Monitoring: Lessons from a Large Scale Nutrition Program in Madagascar”. Policy Research Working Paper 4058, World Bank.

Galiani, S., P. Gertler y E. Schargrodsky (2005). "Water for Life: The Impact of the Privatization of Water Service on Child Mortality". Journal of Political Economy, Vol. 113:83-120.

Gertler, P., S. Martinez, P. Premand, L. Rawlings y C. Vermeersch (2010). Impact Evaluation in Practice, Washington DC: Banco Mundial.

Gertler, P., S. Martinez y P. Celhay (2011). "Impacto del programa Plan Nacer sobre utilización de servicios y resultados sanitarios. Resultados intermedios con datos administrativos de las provincias de Misiones y Tucumán”. Manuscrito no publicado, Banco Mundial.

Imas, L. y R. Ray (2009). The Road to Results: Designing and Conducting Effective Development Evaluations. Washington DC: Banco Mundial. 
Instituto Nacional de Estadísticas y Censos. Sistema de Estadísticas Socio-demográficas (SESD). Área Salud. Argentina.

King, E. M. y J. R. Behrman (2008). "Timing and Duration of Exposure in Evaluations of Social Programs”. Policy Research Working Paper 4686, World Bank.

Meessen, B., J. Kashala y L. Musango (2007). "Output-based payment to boost staff productivity in public health centres: Contracting in Kaburate district, Rwanda". Bulletin of World Health Organization, Vol.85(2):108-115.

Ministerio de Salud de la Nación (2004). "Proyecto de Inversión en Salud Materno Infantil Provincial. Manual Operativo Fase I".

Ministerio de Salud de la Nación (2007). Informe de la Gestión del Plan Nacer a Octubre de 2007. Área Técnica Unidad Ejecutora Central (UEC).

Naciones Unidas (2010). “Objetivos de Desarrollo del Milenio. Informe 2010”. Nueva York.

Organización Panamericana de la Salud (2010). Situación de Salud en la Américas. Indicadores Básicos 2010.

Porto A. (2006). "Transferencias del Plan Nacer en el contexto de las relaciones con las provincias". Proyecto PNUD ARG/04/023 SMI.

Programa de la Naciones Unidas para el Desarrollo (2005). "La cooperación Internacional ante una encrucijada ayuda al desarrollo, comercio y seguridad en un mundo desigual". Informe sobre desarrollo Humano.

Titmuss R, (1943), Poverty and Wealth: A Study of Infant Mortality. Hamish Hamilton Medical Books, London.

Toonen, J., A. Canavan, P. Vergeer y R. Eloviano (2009). "Learning Leassons on implementing performanced Based financing from a multi-country evaluation". Royal Tropical Institute, Development Policy \& Practice, Amsterdam. 
Tabla 1. Principales causas de muerte para menores de 1 año como porcentaje del total de defunciones. Año 2004

\begin{tabular}{lc} 
Principales Causas: & $\%$ \\
\hline Trastornos relacionados con la duración de la gestación & 12,7 \\
Dificultad respiratoria del recién nacido & 11,8 \\
Otras malformaciones congénitas & 10,1 \\
Sepsis bacteriana del recién nacido & 7,8 \\
Malformaciones congénitas del corazón & 7,4 \\
Otras afecciones respiratorias del recién nacido & 6,3 \\
Resto de afecciones perinatales & 5,6 \\
Otras & 38,2 \\
Total & 100,0 \\
\hline
\end{tabular}

Fuente: Elaboración propia en base a "Defunciones de menores de cinco años indicadores seleccionados". Ministerio de Salud de la Nación, Argentina, 2004.

Tabla 2. Defunciones neonatales según criterios de reducibilidad. Año 2004

\begin{tabular}{|c|c|}
\hline Grupos de causas & $\%$ \\
\hline \multicolumn{2}{|c|}{ Reducibles por dianóstico y tratamiento oportuno } \\
\hline En el embarazo & 34,3 \\
\hline En el parto & 10,6 \\
\hline En el recién nacido & 9,9 \\
\hline Otras reducibles & 1,3 \\
\hline Difícilmente reducibles & 40,1 \\
\hline Otras causas & 1,1 \\
\hline Mal definidas & 2,7 \\
\hline Total & 100,0 \\
\hline
\end{tabular}

Fuente: Elaboración propia en base a “Estadísticas Vitales. Información básica 2004”. Ministerio de Salud de la Nación, Argentina, 2004.

Tabla 3. Defunciones post-neonatales según criterios de reducibilidad. Año 2004

\begin{tabular}{lc}
\multicolumn{1}{c}{ Grupos de causas } & $\%$ \\
\hline Reducibles por prevención & 12,4 \\
Reducibles por tratamiento & 9,0 \\
Reducibles por prevención y tratamiento & 25,5 \\
Otras reducibles & 9,8 \\
Difícilmente reducibles & 26,5 \\
Otras causas & 6,8 \\
Mal definidas & 10,0 \\
Total & 100,0 \\
\hline
\end{tabular}

Fuente: Elaboración propia en base a “Estadísticas Vitales. Información básica 2004”. Ministerio de Salud de la Nación. Rep. Argentina. Año 2004. 
Tabla 4. Indicadores socioeconómicos por provincia. Año 2004

\begin{tabular}{|c|c|c|c|c|c|c|}
\hline Provincias & $\begin{array}{c}\text { Muy bajo } \\
\text { peso al } \\
\text { nacer (\%) }\end{array}$ & $\begin{array}{l}\text { Bajo peso al } \\
\text { nacer (\%) }\end{array}$ & $\begin{array}{c}\text { Nacidos } \\
\text { vivos madres } \\
\text { menores a } \\
15 \text { años (\%) }\end{array}$ & $\begin{array}{c}\text { Nacidos } \\
\text { vivos madres } \\
\text { menores a } \\
20 \text { años }(\%)\end{array}$ & $\begin{array}{c}\text { Tasa de } \\
\text { mortalidad } \\
\text { materna (por } \\
\text { diez mil) }\end{array}$ & $\begin{array}{c}\text { Tasa de } \\
\text { mortalidad } \\
\text { infantil (por } \\
\text { mil) }\end{array}$ \\
\hline Región centro & 1,2 & 7,7 & 0,2 & 12,5 & 2,8 & 12,6 \\
\hline Cdad. de Bs. As. & 1,2 & 7,5 & 0,1 & 6,1 & 2,0 & 8,7 \\
\hline Buenos Aires & 1,2 & 8,0 & 0,1 & 11,9 & 2,8 & 13,0 \\
\hline Córdoba & 1,0 & 7,2 & 0,3 & 13,2 & 2,6 & 12,7 \\
\hline Entre Ríos & 1,0 & 7,5 & 0,6 & 16,4 & 2,6 & 15,5 \\
\hline Santa Fe & 1,1 & 7,3 & 0,6 & 16,4 & 3,6 & 12,0 \\
\hline Región Cuyo & 1,3 & 7,6 & 0,3 & 14,5 & 5,3 & 15,0 \\
\hline La Rioja & 1,3 & 7,4 & 0,6 & 17,1 & 13,6 & 18,2 \\
\hline Mendoza & 1,4 & 7,6 & 0,3 & 13,7 & 3,9 & 13,5 \\
\hline San Juan & 1,4 & 7,5 & 0,2 & 13,3 & 4,7 & 16,4 \\
\hline San Luis & 1,1 & 8,0 & 0,5 & 15,3 & 4,6 & 15,2 \\
\hline Región Noroeste & 1,2 & 7,1 & 0,4 & 13,9 & 5,9 & 17,6 \\
\hline Catamarca & 1,0 & 6,8 & 0,5 & 18,4 & 0,0 & 21,8 \\
\hline Jujuy & 1,4 & 6,7 & 0,6 & 16,8 & 13,1 & 17,8 \\
\hline Salta & 1,1 & 6,9 & 0,3 & 15,8 & 4,4 & 15,4 \\
\hline Sgo. del Estero & 0,8 & 7,0 & 0,7 & 17,2 & 7,3 & 13,8 \\
\hline Tucumán & 1,6 & 7,4 & 0,3 & 15,0 & 4,3 & 20,5 \\
\hline Región Noreste & 1,0 & 7,5 & 0,9 & 20,9 & 8,4 & 20,0 \\
\hline Corrientes & 1,1 & 7,7 & 0,7 & 17,7 & 10,4 & 19,3 \\
\hline Chaco & 0,8 & 7,6 & 1,1 & 22,8 & 7,0 & 21,3 \\
\hline Formosa & 1,1 & 7,7 & 0,9 & 20,1 & 11,1 & 25,1 \\
\hline Misiones & 0,9 & 7,2 & 0,8 & 19,3 & 6,7 & 16,6 \\
\hline Reg. Patagónica & 1,1 & 6,7 & 0,5 & 17,0 & 2,2 & 12,2 \\
\hline Chubut & 0,9 & 6,0 & 0,4 & 17,4 & 1,2 & 11,8 \\
\hline La Pampa & 1,1 & 7,5 & 0,6 & 16,5 & 0,0 & 14,6 \\
\hline Neuquén & 1,2 & 6,8 & 0,6 & 16,5 & 3,9 & 11,1 \\
\hline Río Negro & 1,3 & 7,1 & 0,5 & 17,2 & 2,8 & 14,1 \\
\hline Santa Cruz & 0,9 & 6,5 & 0,2 & 15,3 & 0,0 & 11,9 \\
\hline Tierra del Fuego & 1,2 & 5,8 & 0,4 & 13,4 & 4,6 & 4,1 \\
\hline
\end{tabular}

Nota: Se considera muy bajo peso los nacidos con un peso inferior a los 1.500 gramos y bajo peso a los nacidos con un peso inferior a los 2.500 gramos

Fuente: Elaboración propia en base a DEIS Ministerio de Salud. Todos los valores corresponden a 2004. Las estadísticas vitales se asignan según lugar de residencia de la madre.

Tabla 5. Principales causas de muerte para menores de 1 año como porcentaje del total de defunciones por regiones. Año 2004

\begin{tabular}{lcc}
\multicolumn{1}{c}{ Causa } & NOA y NEA & Resto de Argentina \\
\hline Dificultad respiratoria del recién nacido & 14,4 & 10,6 \\
Trastornos relacionados con la duración de la gestación & 11,7 & 13,2 \\
Sepsis bacteriana del recién nacido & 9,2 & 7,0 \\
Otras malformaciones congénitas & 8,2 & 11,0 \\
Otras afecciones respiratorias del recién nacido & 7,6 & 8,5 \\
Resto & 48,9 & 49,7 \\
Total & 100,0 & 100,0 \\
\hline
\end{tabular}

Fuente: Elaboración propia en base a "Defunciones de menores de cinco años indicadores seleccionados". Ministerio de Salud de la Nación. Rep. Argentina. Año 2004. 
Tabla 6. Regresiones caso binario. Tasa de Mortalidad Infantil. Años 2004 y 2007

\begin{tabular}{|c|c|c|c|c|c|c|c|c|}
\hline & (a) & (b) & (c) & (d) & (e) & (f) & (g) & (h) \\
\hline Variables Explicativas & & & & & & & & \\
\hline Tratamiento & $\begin{array}{c}-1,844^{* \star *} \\
(0,633)\end{array}$ & $\begin{array}{c}-1,839^{* * *} \\
(0,633)\end{array}$ & $\begin{array}{c}-1,810^{\star \star \star} \\
(0,638)\end{array}$ & $\begin{array}{c}-1,849^{* \star *} \\
(0,647)\end{array}$ & & $\begin{array}{c}-1,837^{\star \star \star} \\
(0,649)\end{array}$ & & $\begin{array}{c}0,814 \\
(0,579)\end{array}$ \\
\hline Cambio poblacional & & $\begin{array}{c}0,007 \\
(0,009)\end{array}$ & & $\begin{array}{c}0,009 \\
(0,017)\end{array}$ & & $\begin{array}{c}0,009 \\
(0,017)\end{array}$ & & $\begin{array}{l}0,032^{\star *} \\
(0,015)\end{array}$ \\
\hline Cambio en la cantidad de nacidos vivos & & & $\begin{array}{c}0,000 \\
(0,000)\end{array}$ & $\begin{array}{l}-0,000 \\
(0,001)\end{array}$ & & $\begin{array}{l}-0,000 \\
(0,001)\end{array}$ & & $\begin{array}{c}-0,001^{* *} \\
(0,001)\end{array}$ \\
\hline Intensidad inicial de los establecimientos de salud públicos & & & & & $\begin{array}{c}0,305 \\
(0,211)\end{array}$ & $\begin{array}{c}0,228 \\
(0,202)\end{array}$ & & $\begin{array}{c}-0,798^{* * *} \\
(0,251)\end{array}$ \\
\hline Tasa de Mortalidad Infantil inicial & & & & & & & $\begin{array}{c}-0,407^{\star * *} \\
(0,041)\end{array}$ & $\begin{array}{c}-0,450^{\star \star \star *} \\
(0,043)\end{array}$ \\
\hline Constante & $\begin{array}{l}-0,125 \\
(0,344)\end{array}$ & $\begin{array}{l}-0,144 \\
(0,346)\end{array}$ & $\begin{array}{l}-0,113 \\
(0,345)\end{array}$ & $\begin{array}{l}-0,153 \\
(0,363)\end{array}$ & $\begin{array}{c}-0,781^{* * *} \\
(0,297)\end{array}$ & $\begin{array}{l}-0,186 \\
(0,369)\end{array}$ & $\begin{array}{c}4,755^{\star \star \star} \\
(0,578)\end{array}$ & $\begin{array}{c}4,964^{* * *} \\
(0,605)\end{array}$ \\
\hline Efectos Fijos a nivel departamento & $\mathrm{Si}$ & $\mathrm{Si}$ & $\mathrm{Si}$ & $\mathrm{Si}$ & $\mathrm{Si}$ & $\mathrm{Si}$ & $\mathrm{Si}$ & $\mathrm{Si}$ \\
\hline R2 & 0,021 & 0,022 & 0,022 & 0,022 & 0,001 & 0,023 & 0,239 & 0,254 \\
\hline
\end{tabular}

Errores estándar por clusters entre paréntesis.

Nota 1: *** $\mathrm{p}<0,01: * * \mathrm{p}<0,05 ; * \mathrm{p}<0,1$.

Nota 2: La variable dependiente en todas las columnas es el cambio en la tasa de mortalidad infantil entre el año 2004 y 2007 . El tratamiento es una variable binaria que toma el valor uno para los departamentos bajo programa en el año 2007 y cero para cualquier otro caso; el cambio poblacional es, valga la redundancia, el cambio poblacional entre 2004 y 2007; lo mismo vale para el cambio en los nacidos vivos. La intensidad inicial es el cociente entre la población y la cantidad de establecimientos públicos de salud en el año 2004 y la tasa de mortalidad infantil inicial es la que se corresponde con el período base, es decir con el 2004.

Nota 3: En todas las regresiones se consideran aquellos departamentos que modifican su tasa de mortalidad infantil como máximo hasta 15 puntos porcentuales en valor absoluto. 
Tabla 7. Regresiones considerando distintos grados de exposición. Tasa de Mortalidad Infantil. Años 2004 y 2007

\begin{tabular}{|c|c|c|c|c|c|c|c|c|c|}
\hline & (a) & (b) & (c) & (d) & (e) & (f) & (g) & (h) & (i) \\
\hline Variables Explicativas & & & & & & & & & \\
\hline Grado de Exposición al programa & $\begin{array}{c}-2,032^{* *} \\
(0,956)\end{array}$ & $\begin{array}{c}-2,070^{\star *} \\
(0,969)\end{array}$ & $\begin{array}{c}-2,081^{* *} \\
(0,969)\end{array}$ & $\begin{array}{c}-2,082^{\star *} \\
(0,970)\end{array}$ & $\begin{array}{c}-2,122^{\star *} \\
(0,955)\end{array}$ & & $\begin{array}{l}-1,281 \\
(0,948)\end{array}$ & & $\begin{array}{l}-1,369^{*} \\
(0,785)\end{array}$ \\
\hline Cambio en la cantidad de nacidos vivos & & & $\begin{array}{c}0,000 \\
(0,000)\end{array}$ & $\begin{array}{c}0,001 \\
(0,001)\end{array}$ & $\begin{array}{c}0,001 \\
(0,001)\end{array}$ & & $\begin{array}{c}0,000 \\
(0,001)\end{array}$ & & $\begin{array}{l}-0,001^{*} \\
(0,001)\end{array}$ \\
\hline Intensidad inicial de los establecimientos de salud público & & & & & $\begin{array}{c}0,390 \\
(0,272)\end{array}$ & & $\begin{array}{c}0,306 \\
(0,240)\end{array}$ & & $\begin{array}{c}-0,716^{\star \star \star} \\
(0,218)\end{array}$ \\
\hline Plan Nacer ( $=1$ si el departamento está en NOA o NEA) & & & & & & $\begin{array}{c}-1,844^{\star \star \star} \\
(0,633)\end{array}$ & $\begin{array}{r}-1,300^{*} \\
(0,765)\end{array}$ & & $\begin{array}{l}1,391^{\star \star} \\
(0,689)\end{array}$ \\
\hline Tasa de Mortalidad Infantil inicial & & & & & & & & $\begin{array}{c}-0,407^{\star * *} \\
(0,041)\end{array}$ & $\begin{array}{c}-0,451^{\star \star \star} \\
(0,042)\end{array}$ \\
\hline Efectos Fijos a nivel departamento & $\mathrm{Si}$ & $\mathrm{Si}$ & $\mathrm{Si}$ & $\mathrm{Si}$ & $\mathrm{Si}$ & $\mathrm{Si}$ & $\mathrm{Si}$ & $\mathrm{Si}$ & $\mathrm{Si}$ \\
\hline Cantidad de Observaciones & 432 & 432 & 432 & 432 & 432 & 432 & 432 & 432 & 432 \\
\hline R2 & 0,016 & 0,018 & 0,019 & 0,019 & 0,020 & 0,021 & 0,027 & 0,239 & 0,259 \\
\hline
\end{tabular}

Errores estándar por clusters entre paréntesis.

Nota $1: * * * \mathrm{p}<0,01 ; * * \mathrm{p}<0,05 ; * \mathrm{p}<0,1$.

Nota 2: La variable dependiente en todas las columnas es el cambio en la tasa de mortalidad infantil entre el año 2004 y 2007 . El grado de exposición es el cociente entre los establecimiento con convenio relativo a la población del año 2004; el cambio poblacional es, valga la redundancia, el cambio poblacional entre 2004 y 2007 ; lo mismo vale para el cambio en los nacidos vivos. La intensidad inicial es el cociente entre la población y la cantidad de establecimientos públicos de salud en el año 2004 y la tasa de mortalidad infantil inicial es la que se corresponde con el período base, es decir con el 2004. Plan Nacer es una variable binaria que identifica aquellos departamentos que se encuentran en las regiones bajo programa.

Nota 3: En todas las regresiones se consideran aquellos departamentos que modifican su tasa de mortalidad infantil como máximo hasta 15 puntos porcentuales en valor absoluto. 
Tabla 8. Experimento de Falsificación. Años 2001 y 2004

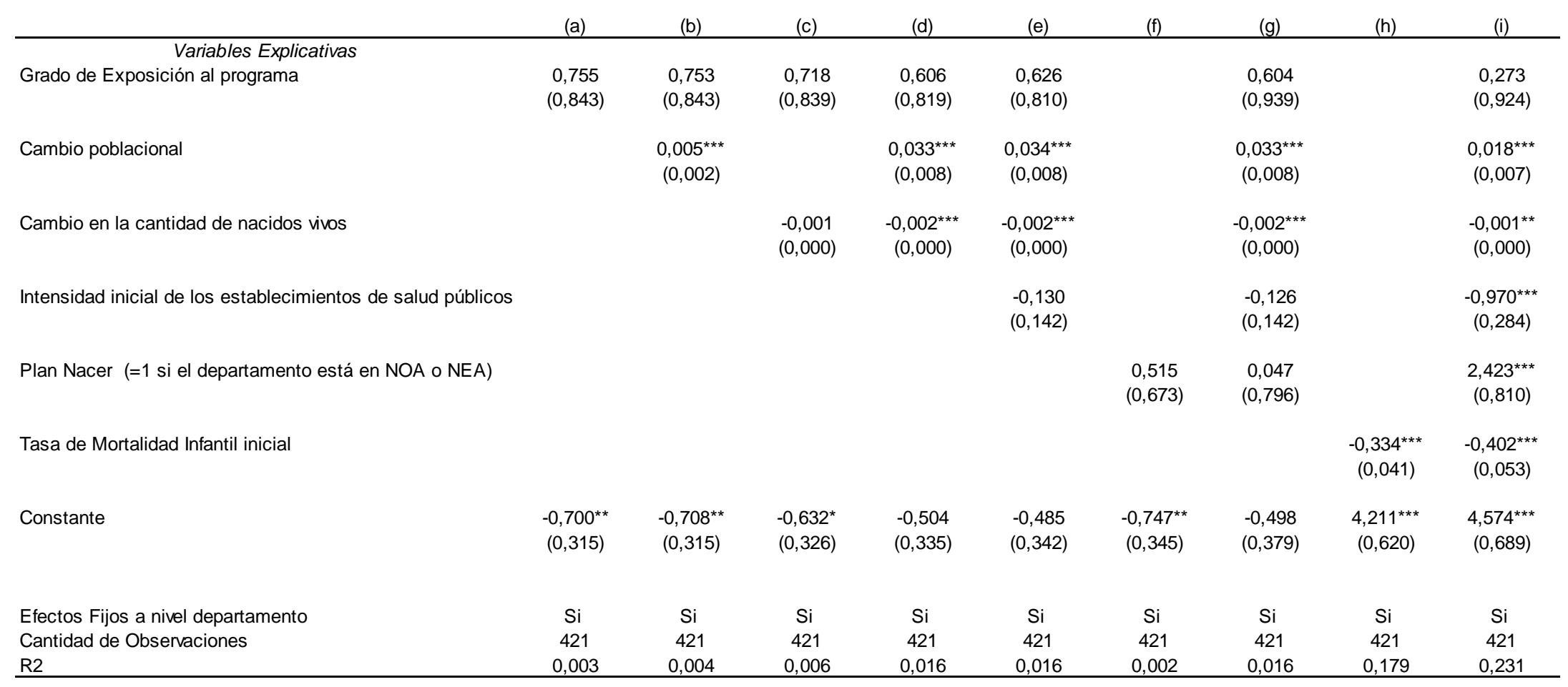

Errores estándar por clusters entre paréntesis

Nota $1: * * * \mathrm{p}<0,01 ; * * \mathrm{p}<0,05 ; * \mathrm{p}<0,1$.

Nota 2: La variable dependiente en todas las columnas es el cambio en la tasa de mortalidad infantil entre el año 2001 y 2004 . El grado de exposición es el cociente entre los establecimiento con convenio relativo a la población del año 2001; el cambio poblacional es, valga la redundancia, el cambio poblacional entre 2001 y 2004 ; lo mismo vale para el cambio en los nacidos vivos. La intensidad inicial es el cociente entre la población y la cantidad de establecimientos públicos de salud en el año 2001 y la tasa de mortalidad infantil inicial es la que se corresponde con el período base, es decir con el 2001. Plan Nacer es una variable binaria que identifica aquellos departamentos que se encuentran en las regiones bajo programa.

Nota 3: En todas las regresiones se consideran aquellos departamentos que modifican su tasa de mortalidad infantil como máximo hasta 15 puntos porcentuales en valor absoluto. 
Tabla 9. Regresiones Tasa de Mortalidad Infantil, en el grado de exposición se considera no solo la intensidad de cada departamento sino también la de los departamentos vecinos. Años 2004 y 2007

\begin{tabular}{|c|c|c|c|c|c|c|c|c|c|c|c|}
\hline & (a) & (b) & (c) & (d) & (e) & (f) & (g) & (h) & (i) & (j) & (k) \\
\hline $\begin{array}{c}\text { Variables Explicativas } \\
\text { Grado de Exposición al programa }\end{array}$ & $\begin{array}{l}-2,032^{* *} \\
(0,956)\end{array}$ & $\begin{array}{l}-2,070^{* *} \\
(0,969)\end{array}$ & $\begin{array}{l}-2,081^{* *} \\
(0,969)\end{array}$ & $\begin{array}{l}-2,082^{* *} \\
(0,970)\end{array}$ & $\begin{array}{l}-2,122^{* *} \\
(0,955)\end{array}$ & & $\begin{array}{l}-1,281 \\
(0,948)\end{array}$ & & $\begin{array}{l}-1,369^{*} \\
(0,785)\end{array}$ & & \\
\hline Cambio poblacional & & $\begin{array}{c}0,008 \\
(0,010)\end{array}$ & & $\begin{array}{l}-0,002 \\
(0,017)\end{array}$ & $\begin{array}{l}-0,001 \\
(0,017)\end{array}$ & & $\begin{array}{c}0,006 \\
(0,017)\end{array}$ & & $\begin{array}{l}0,029^{\star *} \\
(0,015)\end{array}$ & & $\begin{array}{l}0,029^{*} \\
(0,015)\end{array}$ \\
\hline Cambio en la cantidad de nacidos vivos & & & $\begin{array}{c}0,000 \\
(0,000)\end{array}$ & $\begin{array}{c}0,001 \\
(0,001)\end{array}$ & $\begin{array}{c}0,001 \\
(0,001)\end{array}$ & & $\begin{array}{l}0,000 \\
(0,001)\end{array}$ & & $\begin{array}{l}-0,001^{*} \\
(0,001)\end{array}$ & & $\begin{array}{l}-0,001^{*} \\
(0,001)\end{array}$ \\
\hline Intensidad inicial de los establecimientos de salud públic & & & & & $\begin{array}{l}0,390 \\
(0,272)\end{array}$ & & $\begin{array}{l}0,306 \\
(0,240)\end{array}$ & & $\begin{array}{l}-0,716^{* \star *} \\
(0,218)\end{array}$ & & $\begin{array}{l}-0,701^{* * *} \\
(0,214)\end{array}$ \\
\hline Plan Nacer ( $=1$ si el departamento está en NOA o NEA) & & & & & & $\begin{array}{c}-1,844^{\star \star *} \\
(0,633)\end{array}$ & $\begin{array}{l}-1,300^{*} \\
(0,765)\end{array}$ & & $\begin{array}{l}1,391^{* *} \\
(0,689)\end{array}$ & & $\begin{array}{l}1,798^{* *} \\
(0,750)\end{array}$ \\
\hline Tasa de Mortalidad Infantil inicial & & & & & & & & $\begin{array}{c}-0,407^{\star \star \star} \\
(0,041)\end{array}$ & $\begin{array}{l}-0,451^{\star \star \star} \\
(0,042)\end{array}$ & & $\begin{array}{c}-0,448^{\star \star \star} \\
(0,042)\end{array}$ \\
\hline $\begin{array}{l}\text { Grado de Exposición al programa (es la exposición } \\
\text { propia más la suma ponderada de los cinco } \\
\text { departamentos más cercanos) }\end{array}$ & & & & & & & & & & $\begin{array}{r}-1,679^{\star \star *} \\
(0,563)\end{array}$ & $\begin{array}{l}-1,242^{* *} \\
(0,561)\end{array}$ \\
\hline Constante & $\begin{array}{l}-0,474 \\
(0,309)\end{array}$ & $\begin{array}{l}-0,490 \\
(0,310)\end{array}$ & $\begin{array}{l}-0,432 \\
(0,315)\end{array}$ & $\begin{array}{l}-0,421 \\
(0,344)\end{array}$ & $\begin{array}{l}-0,466 \\
(0,349)\end{array}$ & $\begin{array}{l}-0,125 \\
(0,344)\end{array}$ & $\begin{array}{l}-0,180 \\
(0,370)\end{array}$ & $\begin{array}{c}4,755^{* * *} \\
(0,578)\end{array}$ & $\begin{array}{r}4,978^{\star \star \star} \\
(0,600)\end{array}$ & $\begin{array}{l}-0,297 \\
(0,316)\end{array}$ & $\begin{array}{c}4,956^{\star \star *} \\
(0,597)\end{array}$ \\
\hline $\begin{array}{l}\text { Efectos Fijos a nivel departamento } \\
\text { Cantidad de Observaciones } \\
\text { R2 }\end{array}$ & $\begin{array}{c}\mathrm{Si} \\
432 \\
0,016\end{array}$ & $\begin{array}{c}\mathrm{Si} \\
432 \\
0,018\end{array}$ & $\begin{array}{c}\mathrm{Si} \\
432 \\
0,019\end{array}$ & $\begin{array}{c}\mathrm{Si} \\
432 \\
0,019\end{array}$ & $\begin{array}{c}\mathrm{Si} \\
432 \\
0,020\end{array}$ & $\begin{array}{c}\mathrm{Si} \\
432 \\
0,021\end{array}$ & $\begin{array}{c}\mathrm{Si} \\
432 \\
0,027\end{array}$ & $\begin{array}{c}\mathrm{Si} \\
432 \\
0,239\end{array}$ & $\begin{array}{c}\mathrm{Si} \\
432 \\
0,259\end{array}$ & $\begin{array}{c}\mathrm{Si} \\
432 \\
0,029\end{array}$ & $\begin{array}{c}\mathrm{Si} \\
432 \\
0,264\end{array}$ \\
\hline
\end{tabular}

Errores estándar por clusters entre paréntesis

Nota $1: * * * \mathrm{p}<0,01 ; * * \mathrm{p}<0,05 ; * \mathrm{p}<0,1$.

Nota 2: La variable dependiente en todas las columnas es el cambio en la tasa de mortalidad infantil entre el año 2004 y 2007 . El grado de exposición es el cociente entre los establecimiento con convenio relativo a la población del año 2004; la segunda alternativa considera el grado de exposición de un departamento como el propio grado de exposición más la suma ponderada del grado de exposición de los cinco departamentos más cercanos. El cambio poblacional es, valga la redundancia, el cambio poblacional entre 2004 y 2007; lo mismo vale para el cambio en los nacidos vivos. La intensidad inicial es el cociente entre la población y la cantidad de establecimientos públicos de salud en el año 2004 y la tasa de mortalidad infantil inicial es la que se corresponde con el período base, es decir con el 2004. Plan Nacer es una variable binaria que identifica aquellos departamentos que se encuentran en las regiones bajo programa.

Nota 3: En todas las regresiones se consideran aquellos departamentos que modifican su tasa de mortalidad infantil como máximo hasta 15 puntos porcentuales en valor absoluto. 
Tabla 10. Regresiones Tasa de Mortalidad Neonatal. Años 2004 y 2007

\begin{tabular}{|c|c|c|c|c|c|c|c|c|c|c|c|}
\hline & (a) & (b) & (c) & (d) & (e) & (f) & (g) & (h) & (i) & (j) & $(\mathrm{k})$ \\
\hline $\begin{array}{l}\text { Variables Explicativas } \\
\text { Grado de Exposición al programa }\end{array}$ & $\begin{array}{c}-1,266^{\star \star} \\
(0,619)\end{array}$ & $\begin{array}{c}-1,287^{* *} \\
(0,620)\end{array}$ & $\begin{array}{c}-1,306^{\star \star} \\
(0,620)\end{array}$ & $\begin{array}{c}-1,315^{\star *} \\
(0,620)\end{array}$ & $\begin{array}{c}-1,364^{\star \star} \\
(0,623)\end{array}$ & & $\begin{array}{l}-1,089 \\
(0,727)\end{array}$ & & $\begin{array}{l}-0,897 \\
(0,608)\end{array}$ & & \\
\hline Cambio poblacional & & $\begin{array}{c}0,008 \\
(0,009)\end{array}$ & & $\begin{array}{l}-0,014 \\
(0,015)\end{array}$ & $\begin{array}{l}-0,013 \\
(0,015)\end{array}$ & & $\begin{array}{l}-0,011 \\
(0,015)\end{array}$ & & $\begin{array}{c}0,018 \\
(0,011)\end{array}$ & & $\begin{array}{c}0,018 \\
(0,011)\end{array}$ \\
\hline Cambio en la cantidad de nacidos vivos & & & $\begin{array}{c}0,001 \\
(0,000)\end{array}$ & $\begin{array}{l}0,001^{*} \\
(0,001)\end{array}$ & $\begin{array}{l}0,001^{\star} \\
(0,001)\end{array}$ & & $\begin{array}{c}0,001 \\
(0,001)\end{array}$ & & $\begin{array}{l}-0,001 \\
(0,001)\end{array}$ & & $\begin{array}{l}-0,001 \\
(0,001)\end{array}$ \\
\hline Intensidad inicial de los establecimientos de salud público: & & & & & $\begin{array}{c}0,245 \\
(0,189)\end{array}$ & & $\begin{array}{c}0,198 \\
(0,176)\end{array}$ & & $\begin{array}{c}-0,740^{\star \star \star} \\
(0,275)\end{array}$ & & $\begin{array}{c}-0,739^{\star \star \star} \\
(0,279)\end{array}$ \\
\hline Plan Nacer ( $=1$ si el departamento está en NOA o NEA) & & & & & & $\begin{array}{c}-1,108^{\star *} \\
(0,547)\end{array}$ & $\begin{array}{l}-0,527 \\
(0,645)\end{array}$ & & $\begin{array}{l}0,975^{\star} \\
(0,555)\end{array}$ & & $\begin{array}{l}1,150^{*} \\
(0,612)\end{array}$ \\
\hline Tasa de Mortalidad Neonatal inicial & & & & & & & & $\begin{array}{c}-0,510^{* \star \star} \\
(0,042)\end{array}$ & $\begin{array}{c}-0,544^{* \star \star} \\
(0,045)\end{array}$ & & $\begin{array}{c}-0,541^{\star \star \star} \\
(0,045)\end{array}$ \\
\hline $\begin{array}{l}\text { Grado de Exposición al programa (es la exposición } \\
\text { propia más la suma ponderada de los cinco } \\
\text { departamentos más cercanos) }\end{array}$ & & & & & & & & & & $\begin{array}{r}-1,041^{\star \star \star} \\
(0,392)\end{array}$ & $\begin{array}{l}-0,687 \\
(0,425)\end{array}$ \\
\hline Constante & $\begin{array}{c}-0,635^{\star *} \\
(0,266)\end{array}$ & $\begin{array}{c}-0,650^{\star *} \\
(0,266)\end{array}$ & $\begin{array}{c}-0,590^{\star *} \\
(0,270)\end{array}$ & $\begin{array}{l}-0,507^{*} \\
(0,294)\end{array}$ & $\begin{array}{l}-0,536^{*} \\
(0,299)\end{array}$ & $\begin{array}{l}-0,445 \\
(0,303)\end{array}$ & $\begin{array}{l}-0,409 \\
(0,326)\end{array}$ & $\begin{array}{c}3,700^{\star \star \star} \\
(0,395)\end{array}$ & $\begin{array}{c}3,809^{\star \star \star} \\
(0,434)\end{array}$ & $\begin{array}{l}-0,517^{*} \\
(0,273)\end{array}$ & $\begin{array}{c}3,790^{* * *} \\
(0,434)\end{array}$ \\
\hline Efectos Fijos a nivel departamento & $\mathrm{Si}$ & $\mathrm{Si}$ & $\mathrm{Si}$ & $\mathrm{Si}$ & $\mathrm{Si}$ & $\mathrm{Si}$ & $\mathrm{Si}$ & $\mathrm{Si}$ & $\mathrm{Si}$ & $\mathrm{Si}$ & $\mathrm{Si}$ \\
\hline Cantidad de Observaciones & 468 & 468 & 468 & 468 & 468 & 468 & 468 & 468 & 468 & 468 & 468 \\
\hline $\mathrm{R} 2$ & 0,011 & 0,013 & 0,015 & 0,016 & 0,017 & 0,009 & 0,018 & 0,281 & 0,298 & 0,017 & 0,299 \\
\hline
\end{tabular}

Errores estándar por clusters entre paréntesis

Nota $1: * * * \mathrm{p}<0,01 ; * * \mathrm{p}<0,05 ; * \mathrm{p}<0,1$.

Nota 2: La variable dependiente en todas las columnas es el cambio en la tasa de mortalidad neonatal entre el año 2004 y 2007 . El grado de exposición es el cociente entre los establecimiento con convenio relativo a la población del año 2004; la segunda alternativa considera el grado de exposición de un departamento como el propio grado de exposición más la suma ponderada del grado de exposición de los cinco departamentos más cercanos. El cambio poblacional es, valga la redundancia, el cambio poblacional entre 2004 y 2007; lo mismo vale para el cambio en los nacidos vivos. La intensidad inicial es el cociente entre la población y la cantidad de establecimientos públicos de salud en el año 2004 y la tasa de mortalidad neonatal inicial es la que se corresponde con el período base, es decir con el 2004. Plan Nacer es una variable binaria que identifica aquellos departamentos que se encuentran en las regiones bajo programa.

Nota 3: En todas las regresiones se consideran aquellos departamentos que modifican su tasa de mortalidad neonatal como máximo hasta 15 puntos porcentuales en valor absoluto. 
Tabla 11. Regresiones Tasa de Mortalidad Post Neonatal. Años 2004 y 2007

\begin{tabular}{|c|c|c|c|c|c|c|c|c|c|c|c|}
\hline & (a) & (b) & (c) & (d) & (e) & (f) & (g) & (h) & (i) & (j) & (k) \\
\hline $\begin{array}{c}\text { Variables Explicativas } \\
\text { Grado de Exposición al programa }\end{array}$ & $\begin{array}{c}-1,464^{* *} \\
(0,614)\end{array}$ & $\begin{array}{c}-1,470^{* *} \\
(0,618)\end{array}$ & $\begin{array}{c}-1,459^{* *} \\
(0,616)\end{array}$ & $\begin{array}{c}-1,455^{\star *} \\
(0,619)\end{array}$ & $\begin{array}{c}-1,460^{* *} \\
(0,616)\end{array}$ & & $\begin{array}{l}-1,140^{*} \\
(0,661)\end{array}$ & & $\begin{array}{c}-1,359^{\star \star *} \\
(0,402)\end{array}$ & & \\
\hline Cambio poblacional & & $\begin{array}{c}0,001 \\
(0,002)\end{array}$ & & $\begin{array}{l}0,014^{*} \\
(0,008)\end{array}$ & $\begin{array}{l}0,015^{*} \\
(0,008)\end{array}$ & & $\begin{array}{l}0,017^{*} \\
(0,009)\end{array}$ & & $\begin{array}{l}0,016^{*} \\
(0,009)\end{array}$ & & $\begin{array}{l}0,016^{*} \\
(0,009)\end{array}$ \\
\hline Cambio en la cantidad de nacidos vivos & & & $\begin{array}{l}-0,000 \\
(0,000)\end{array}$ & $\begin{array}{l}-0,001 \\
(0,000)\end{array}$ & $\begin{array}{l}-0,001 \\
(0,000)\end{array}$ & & $\begin{array}{l}-0,001^{*} \\
(0,001)\end{array}$ & & $\begin{array}{l}-0,001^{*} \\
(0,000)\end{array}$ & & $\begin{array}{l}-0,001^{*} \\
(0,000)\end{array}$ \\
\hline Intensidad inicial de los establecimientos de salud públicos & & & & & $\begin{array}{c}0,055 \\
(0,091)\end{array}$ & & $\begin{array}{c}0,025 \\
(0,088)\end{array}$ & & $\begin{array}{c}-0,600^{\star * \star} \\
(0,227)\end{array}$ & & $\begin{array}{c}-0,599^{* \star *} \\
(0,228)\end{array}$ \\
\hline Plan Nacer ( $=1$ si el departamento está en NOA o NEA) & & & & & & $\begin{array}{l}-0,846^{*} \\
(0,480)\end{array}$ & $\begin{array}{l}-0,464 \\
(0,577)\end{array}$ & & $\begin{array}{l}1,683^{\star \star \star} \\
(0,465)\end{array}$ & & $\begin{array}{r}1,959^{\star \star \star} \\
(0,500)\end{array}$ \\
\hline Tasa de Mortalidad Post Neonatal inicial & & & & & & & & $\begin{array}{c}-0,622^{\star \star \star} \\
(0,054)\end{array}$ & $\begin{array}{c}-0,679^{* \star *} \\
(0,056)\end{array}$ & & $\begin{array}{c}-0,673^{\star \star \star} \\
(0,056)\end{array}$ \\
\hline $\begin{array}{l}\text { Grado de Exposición al programa (es la exposición propia } \\
\text { más la suma ponderada de los cinco departamentos más } \\
\text { cercanos) }\end{array}$ & & & & & & & & & & $\begin{array}{l}-1,183^{\star \star \star} \\
(0,342)\end{array}$ & $\begin{array}{l}-1,060^{* \star \star} \\
(0,285)\end{array}$ \\
\hline Constante & $\begin{array}{c}0,078 \\
(0,220)\end{array}$ & $\begin{array}{c}0,076 \\
(0,220)\end{array}$ & $\begin{array}{c}0,074 \\
(0,221)\end{array}$ & $\begin{array}{l}-0,005 \\
(0,244)\end{array}$ & $\begin{array}{l}-0,012 \\
(0,249)\end{array}$ & $\begin{array}{c}0,166 \\
(0,240)\end{array}$ & $\begin{array}{c}0,088 \\
(0,260)\end{array}$ & $\begin{array}{c}2,829^{\star \star *} \\
(0,265)\end{array}$ & $\begin{array}{c}2,704^{\star \star \star} \\
(0,280)\end{array}$ & $\begin{array}{c}0,202 \\
(0,223)\end{array}$ & $\begin{array}{c}2,688^{\star \star *} \\
(0,281)\end{array}$ \\
\hline Efectos Fijos a nivel departamento & $\mathrm{Si}$ & $\mathrm{Si}$ & $\mathrm{Si}$ & $\mathrm{Si}$ & $\mathrm{Si}$ & $\mathrm{Si}$ & $\mathrm{Si}$ & $\mathrm{Si}$ & $\mathrm{Si}$ & & \\
\hline $\begin{array}{l}\text { Cantidad de Observaciones } \\
\text { R2 }\end{array}$ & $\begin{array}{c}487 \\
0.013\end{array}$ & $\begin{array}{c}487 \\
0.013\end{array}$ & $\begin{array}{c}487 \\
0.013\end{array}$ & $\begin{array}{c}487 \\
0.014\end{array}$ & $\begin{array}{c}487 \\
0.014\end{array}$ & $\begin{array}{c}487 \\
0.007\end{array}$ & $\begin{array}{c}487 \\
0.016\end{array}$ & $\begin{array}{c}487 \\
0.340\end{array}$ & $\begin{array}{c}487 \\
0.371\end{array}$ & $\begin{array}{c}487 \\
0.023\end{array}$ & $\begin{array}{c}487 \\
0.374\end{array}$ \\
\hline
\end{tabular}

Errores estándar por clusters entre paréntesis

Nota $1: * * * \mathrm{p}<0,01 ; * * \mathrm{p}<0,05 ; * \mathrm{p}<0,1$

Nota 2: La variable dependiente en todas las columnas es el cambio en la tasa de mortalidad post neonatal entre el año 2004 y 2007 . El grado de exposición es el cociente entre los establecimiento con convenio relativo a la población del año 2004; la segunda alternativa considera el grado de exposición de un departamento como el propio grado de exposición más la suma ponderada del grado de exposición de los cinco departamentos más cercanos. El cambio poblacional es, valga la redundancia, el cambio poblacional entre 2004 y 2007; lo mismo vale para el cambio en los nacidos vivos. La intensidad inicial es el cociente entre la población y la cantidad de establecimientos públicos de salud en el año 2004 y la tasa de mortalidad post neonatal inicial es la que se corresponde con el período base, es decir con el 2004 . Plan Nacer es una variable binaria que identifica aquellos departamentos que se encuentran en las regiones bajo programa.

Nota 3: En todas las regresiones se consideran aquellos departamentos que modifican su tasa de mortalidad post neonatal como máximo hasta 15 puntos porcentuales en valor absoluto. 
Tabla 12. Regresiones Tasa de Mortalidad Neonatal. Experimento de Falsificación Años 2001 y 2004

\begin{tabular}{|c|c|c|c|c|c|c|c|c|c|c|c|}
\hline & (a) & (b) & (c) & (d) & (e) & (f) & (g) & (h) & (i) & (j) & $(\mathrm{k})$ \\
\hline $\begin{array}{l}\text { Variables Explicativas } \\
\text { Grado de Exposición al programa }\end{array}$ & $\begin{array}{c}0,891 \\
(0,707)\end{array}$ & $\begin{array}{c}0,891 \\
(0,708)\end{array}$ & $\begin{array}{c}0,868 \\
(0,710)\end{array}$ & $\begin{array}{c}0,811 \\
(0,715)\end{array}$ & $\begin{array}{c}0,811 \\
(0,709)\end{array}$ & & $\begin{array}{c}0,900 \\
(0,799)\end{array}$ & & $\begin{array}{c}0,694 \\
(0,657)\end{array}$ & & \\
\hline Cambio poblacional & & $\begin{array}{c}0,003 \\
(0,006)\end{array}$ & & $\begin{array}{l}0,024^{\star *} \\
(0,009)\end{array}$ & $\begin{array}{l}0,024^{* *} \\
(0,009)\end{array}$ & & $\begin{array}{l}0,024^{\star *} \\
(0,009)\end{array}$ & & $\begin{array}{c}0,009 \\
(0,007)\end{array}$ & & $\begin{array}{c}0,009 \\
(0,007)\end{array}$ \\
\hline Cambio en la cantidad de nacidos vivos & & & $\begin{array}{l}-0,000 \\
(0,000)\end{array}$ & $\begin{array}{c}-0,001^{\star \star \star} \\
(0,000)\end{array}$ & $\begin{array}{c}-0,001^{\star \star \star} \\
(0,000)\end{array}$ & & $\begin{array}{c}-0,001^{* \star *} \\
(0,000)\end{array}$ & & $\begin{array}{l}-0,000 \\
(0,000)\end{array}$ & & $\begin{array}{l}-0,000 \\
(0,000)\end{array}$ \\
\hline Intensidad inicial de los establecimientos de salud públicos & & & & & $\begin{array}{l}-0,001 \\
(0,124)\end{array}$ & & $\begin{array}{l}-0,022 \\
(0,121)\end{array}$ & & $\begin{array}{c}-0,726^{* \star *} \\
(0,201)\end{array}$ & & $\begin{array}{c}-0,721^{* \star *} \\
(0,205)\end{array}$ \\
\hline Plan Nacer ( $=1$ si el departamento está en NOA o NEA) & & & & & & $\begin{array}{c}0,321 \\
(0,588)\end{array}$ & $\begin{array}{l}-0,231 \\
(0,673)\end{array}$ & & $\begin{array}{l}1,318^{\star *} \\
(0,585)\end{array}$ & & $\begin{array}{l}1,259^{\star *} \\
(0,594)\end{array}$ \\
\hline Tasa de Mortalidad Neonatal inicial & & & & & & & & $\begin{array}{c}-0,482^{\star \star \star} \\
(0,044)\end{array}$ & $\begin{array}{c}-0,519^{\star * \star} \\
(0,051)\end{array}$ & & $\begin{array}{c}-0,519^{\star \star *} \\
(0,051)\end{array}$ \\
\hline \multirow[t]{2}{*}{$\begin{array}{l}\text { Grado de Exposición al programa (es la exposición propia } \\
\text { más la suma ponderada de los cinco departamentos más } \\
\text { cercanos) }\end{array}$} & & & & & & & & & & 0,589 & 0,477 \\
\hline & & & & & & & & & & $(0,438)$ & $(0,421)$ \\
\hline Constante & $\begin{array}{l}-0,491^{*} \\
(0,283)\end{array}$ & $\begin{array}{l}-0,496^{*} \\
(0,283)\end{array}$ & $\begin{array}{l}-0,437 \\
(0,291)\end{array}$ & $\begin{array}{l}-0,352 \\
(0,301)\end{array}$ & $\begin{array}{l}-0,352 \\
(0,306)\end{array}$ & $\begin{array}{l}-0,448 \\
(0,320)\end{array}$ & $\begin{array}{l}-0,281 \\
(0,352)\end{array}$ & $\begin{array}{c}4,125^{\star \star \star} \\
(0,441)\end{array}$ & $\begin{array}{c}4,042^{\star \star \star} \\
(0,471)\end{array}$ & $\begin{array}{l}-0,536^{*} \\
(0,290)\end{array}$ & $\begin{array}{c}4,021^{\star \star \star} \\
(0,472)\end{array}$ \\
\hline Efectos Fijos a nivel departamento & $\mathrm{Si}$ & $\mathrm{Si}$ & $\mathrm{Si}$ & $\mathrm{Si}$ & $\mathrm{Si}$ & $\mathrm{Si}$ & $\mathrm{Si}$ & $\mathrm{Si}$ & $\mathrm{Si}$ & $\mathrm{Si}$ & $\mathrm{Si}$ \\
\hline Cantidad de Observaciones & 460 & 460 & 460 & 460 & 460 & 460 & 460 & 460 & 460 & 460 & 460 \\
\hline $\mathrm{R} 2$ & 0,007 & 0,008 & 0,009 & 0,014 & 0,014 & 0,001 & 0,015 & 0,264 & 0,293 & 0,007 & 0,293 \\
\hline
\end{tabular}

Errores estándar por clusters entre paréntesis

Nota $1: * * * \mathrm{p}<0,01 ; * * \mathrm{p}<0,05 ; * \mathrm{p}<0,1$.

Nota 2: La variable dependiente en todas las columnas es el cambio en la tasa de mortalidad neonatal entre el año 2001 y 2004 . El grado de exposición es el cociente entre los establecimiento con convenio relativo a la población del año 2001; la segunda alternativa considera el grado de exposición de un departamento como el propio grado de exposición más la suma ponderada del grado de exposición de los cinco departamentos más cercanos. El cambio poblacional es, valga la redundancia, el cambio poblacional entre 2001 y 2004; lo mismo vale para el cambio en los nacidos vivos. La intensidad inicial es el cociente entre la población y la cantidad de establecimientos públicos de salud en el año 2001 y la tasa de mortalidad neonatal inicial es la que se corresponde con el período base, es decir con el 2001. Plan Nacer es una variable binaria que identifica aquellos departamentos que se encuentran en las regiones bajo programa.

Nota 3: En todas las regresiones se consideran aquellos departamentos que modifican su tasa de mortalidad neonatal como máximo hasta 15 puntos porcentuales en valor absoluto. 
Tabla 13. Regresiones Tasa de Mortalidad Post Neonatal. Experimento de Falsificación. Años 2001 y 2004

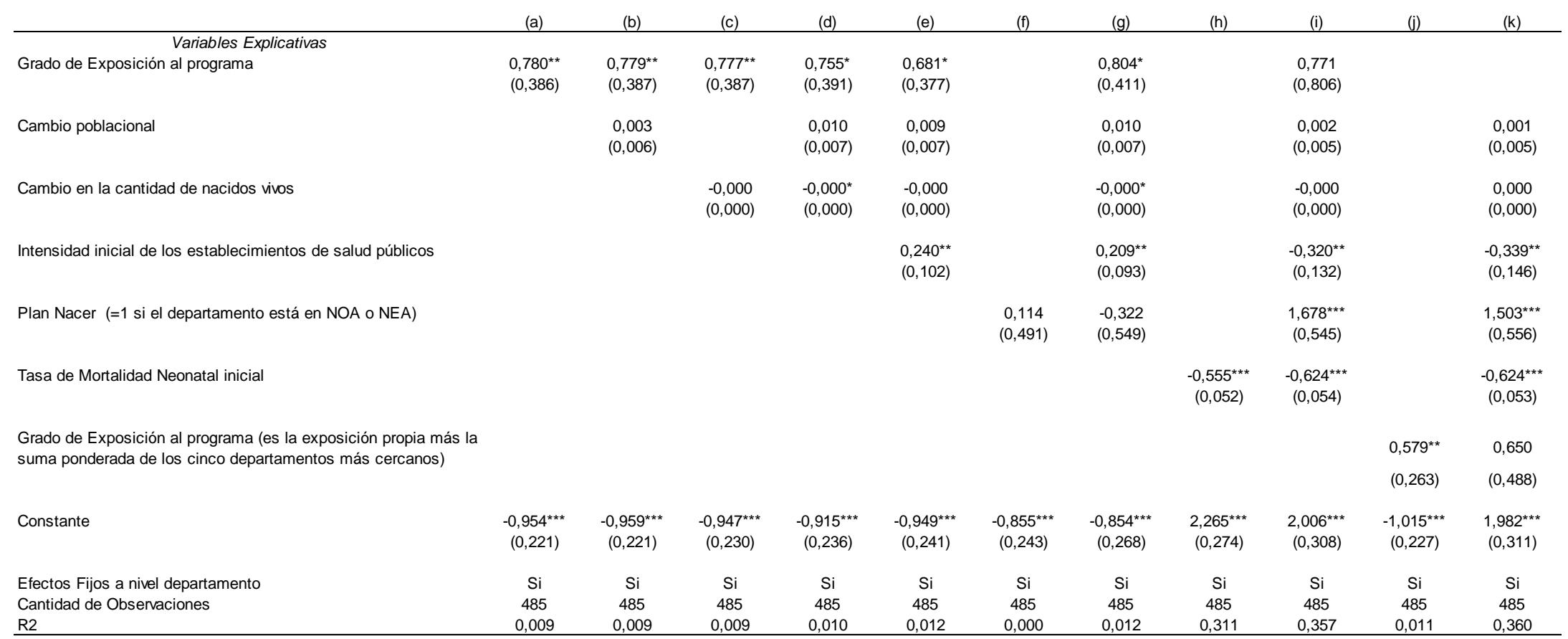

Errores estándar por clusters entre paréntesis

Nota $1: * * * \mathrm{p}<0.01 ; * * \mathrm{p}<0,05 ; * \mathrm{p}<0,1$.

Nota 2: La variable dependiente en todas las columnas es el cambio en la tasa de mortalidad post neonatal entre el año 2001 y 2004 . El grado de exposición es el cociente entre los establecimiento con convenio relativo a la población del año 2001; la segunda alternativa considera el grado de exposición de un departamento como el propio grado de exposición más la suma ponderada del grado de exposición de los cinco departamentos más cercanos. El cambio poblacional es, valga la redundancia, el cambio poblacional entre 2001 y 2004; lo mismo vale para el cambio en los nacidos vivos. La intensidad inicial es el cociente entre la población y la cantidad de establecimientos públicos de salud en el año 2001 y la tasa de mortalidad post neonatal inicial es la que se corresponde con el período base, es decir con el 2001. Plan Nacer es una variable binaria que identifica aquellos departamentos que se encuentran en las regiones bajo programa.

Nota 3: En todas las regresiones se consideran aquellos departamentos que modifican su tasa de mortalidad post neonatal como máximo hasta 15 puntos porcentuales en valor absoluto. 
Tabla 14. Regresiones Tasa de Mortalidad Materna. Años 2004 y 2007

\begin{tabular}{|c|c|c|c|c|c|c|c|c|c|c|c|}
\hline & (a) & (b) & (c) & (d) & (e) & (f) & (g) & (h) & (i) & (j) & (k) \\
\hline $\begin{array}{l}\text { Variables Explicativas } \\
\text { Grado de Exposición al programa }\end{array}$ & $\begin{array}{l}-0,104 \\
(0,114)\end{array}$ & $\begin{array}{l}-0,103 \\
(0,115)\end{array}$ & $\begin{array}{l}-0,101 \\
(0,115)\end{array}$ & $\begin{array}{l}-0,101 \\
(0,115)\end{array}$ & $\begin{array}{l}-0,104 \\
(0,119)\end{array}$ & & $\begin{array}{l}-0,007 \\
(0,097)\end{array}$ & & $\begin{array}{l}-0,116 \\
(0,079)\end{array}$ & & \\
\hline Cambio poblacional & & $\begin{array}{l}-0,000 \\
(0,001)\end{array}$ & & $\begin{array}{l}0,001 \\
(0,003)\end{array}$ & $\begin{array}{l}0,001 \\
(0,003)\end{array}$ & & $\begin{array}{l}0,002 \\
(0,003)\end{array}$ & & $\begin{array}{l}0,003 \\
(0,003)\end{array}$ & & $\begin{array}{c}0,003 \\
(0,003)\end{array}$ \\
\hline Cambio en la cantidad de nacidos vivos & & & $\begin{array}{l}-0,000 \\
(0,000)\end{array}$ & $\begin{array}{l}-0,000 \\
(0,000)\end{array}$ & $\begin{array}{l}-0,000 \\
(0,000)\end{array}$ & & $\begin{array}{l}-0,000 \\
(0,000)\end{array}$ & & $\begin{array}{l}-0,000 \\
(0,000)\end{array}$ & & $\begin{array}{l}-0,000 \\
(0,000)\end{array}$ \\
\hline Intensidad inicial de los establecimientos de salud públicos & & & & & $\begin{array}{c}0,016 \\
(0,033)\end{array}$ & & $\begin{array}{l}0,001 \\
(0,026)\end{array}$ & & $\begin{array}{c}-0,103^{\star *} \\
(0,043)\end{array}$ & & $\begin{array}{c}-0,109^{\star *} \\
(0,047)\end{array}$ \\
\hline Plan Nacer ( $=1$ si el departamento está en NOA ○ NEA) & & & & & & $\begin{array}{l}-0,168 \\
(0,177)\end{array}$ & $\begin{array}{l}-0,178 \\
(0,190)\end{array}$ & & $\begin{array}{l}0,310^{*} \\
(0,163)\end{array}$ & & $\begin{array}{l}0,303^{*} \\
(0,181)\end{array}$ \\
\hline Tasa de Mortalidad Materna inicial & & & & & & & & $\begin{array}{c}-0,901^{\star \star *} \\
(0,075)\end{array}$ & $\begin{array}{c}-0,935^{\star \star *} \\
(0,079)\end{array}$ & & $\begin{array}{c}-0,935^{\star \star \star} \\
(0,080)\end{array}$ \\
\hline $\begin{array}{l}\text { Grado de Exposición al programa (es la exposición propia más } \\
\text { la suma ponderada de los cinco departamentos más cercanos) }\end{array}$ & & & & & & & & & & $-0,047$ & $-0,053$ \\
\hline & & & & & & & & & & $(0,082)$ & $(0,077)$ \\
\hline Efectos Fijos a nivel departamento & $\mathrm{Si}$ & $\mathrm{Si}$ & $\mathrm{Si}$ & $\mathrm{Si}$ & $\mathrm{Si}$ & $\mathrm{Si}$ & $\mathrm{Si}$ & $\mathrm{Si}$ & $\mathrm{Si}$ & $\mathrm{Si}$ & $\mathrm{Si}$ \\
\hline Cantidad de Observaciones & 507 & 507 & 507 & 507 & 507 & 507 & 507 & 507 & 507 & 507 & 507 \\
\hline R2 & 0,001 & 0,001 & 0,001 & 0,001 & 0,001 & 0,002 & 0,003 & 0,299 & 0,308 & 0,000 & 0,307 \\
\hline
\end{tabular}

Errores estándar por clusters entre paréntesis

Nota $1: * * * \mathrm{p}<0,01 ; * * \mathrm{p}<0,05 ; * \mathrm{p}<0,1$

Nota 2: La variable dependiente en todas las columnas es el cambio en la tasa de mortalidad materna entre el año 2004 y 2007 . El grado de exposición es el cociente entre los establecimiento con convenio relativo a la población del año 2004; la segunda alternativa considera el grado de exposición de un departamento como el propio grado de exposición más la suma ponderada del grado de exposición de los cinco departamentos más cercanos. El cambio poblacional es, valga la redundancia, el cambio poblacional entre 2004 y 2007; lo mismo vale para el cambio en los nacidos vivos. La intensidad inicial es el cociente entre la población y la cantidad de establecimientos públicos de salud en el año 2004 y la tasa de mortalidad materna inicial es la que se corresponde con el período base, es decir con el 2004 . Plan Nacer es una variable binaria que identifica aquellos departamentos que se encuentran en las regiones bajo programa.

Nota 3: En todas las regresiones se consideran aquellos departamentos que modifican su tasa de mortalidad maternal como máximo hasta 15 puntos porcentuales en valor absoluto. 
Tabla 15. Regresiones Tasa de Mortalidad Materna Experimento de Falsificación Años 2001y 2004

\begin{tabular}{|c|c|c|c|c|c|c|c|c|c|c|c|}
\hline & (a) & (b) & (c) & (d) & (e) & (f) & (g) & (h) & (i) & (j) & (k) \\
\hline $\begin{array}{l}\text { Variables Explicativas } \\
\text { Grado de Exposición al programa }\end{array}$ & $\begin{array}{l}-0,209 \\
(0,276)\end{array}$ & $\begin{array}{l}-0,209 \\
(0,276)\end{array}$ & $\begin{array}{l}-0,205 \\
(0,276)\end{array}$ & $\begin{array}{l}-0,207 \\
(0,277)\end{array}$ & $\begin{array}{l}-0,205 \\
(0,278)\end{array}$ & & $\begin{array}{l}-0,141 \\
(0,316)\end{array}$ & & $\begin{array}{l}-0,091 \\
(0,064)\end{array}$ & & \\
\hline Cambio poblacional & & $\begin{array}{l}0,001^{\star *} \\
(0,001)\end{array}$ & & $\begin{array}{c}0,001 \\
(0,001)\end{array}$ & $\begin{array}{c}0,001 \\
(0,001)\end{array}$ & & $\begin{array}{c}0,001 \\
(0,001)\end{array}$ & & $\begin{array}{c}0,001 \\
(0,001)\end{array}$ & & $\begin{array}{c}0,001 \\
(0,001)\end{array}$ \\
\hline Cambio en la cantidad de nacidos vivos & & & $\begin{array}{c}0,000 \\
(0,000)\end{array}$ & $\begin{array}{c}0,000 \\
(0,000)\end{array}$ & $\begin{array}{c}0,000 \\
(0,000)\end{array}$ & & $\begin{array}{c}0,000 \\
(0,000)\end{array}$ & & $\begin{array}{l}-0,000 \\
(0,000)\end{array}$ & & $\begin{array}{l}-0,000 \\
(0,000)\end{array}$ \\
\hline Intensidad inicial de los establecimientos de salud públicos & & & & & $\begin{array}{l}-0,008 \\
(0,030)\end{array}$ & & $\begin{array}{l}-0,018 \\
(0,031)\end{array}$ & & $\begin{array}{c}-0,081^{* * *} \\
(0,028)\end{array}$ & & $\begin{array}{c}-0,072^{* * *} \\
(0,026)\end{array}$ \\
\hline Plan Nacer ( $=1$ si el departamento está en NOA o NEA) & & & & & & $\begin{array}{l}-0,198 \\
(0,180)\end{array}$ & $\begin{array}{l}-0,133 \\
(0,207)\end{array}$ & & $\begin{array}{c}0,481^{* \star *} \\
(0,114)\end{array}$ & & $\begin{array}{c}0,539^{\star * \star} \\
(0,128)\end{array}$ \\
\hline Tasa de Mortalidad Materna inicial & & & & & & & & $\begin{array}{c}-0,929 * \star * \\
(0,049)\end{array}$ & $\begin{array}{c}-0,960^{\star \star *} \\
(0,044)\end{array}$ & & $\begin{array}{c}-0,960^{\star \star \star} \\
(0,044)\end{array}$ \\
\hline \multirow[t]{2}{*}{$\begin{array}{l}\text { Grado de Exposición al programa (es la exposición propia } \\
\text { más la suma ponderada de los cinco departamentos más } \\
\text { cercanos) }\end{array}$} & & & & & & & & & & $-0,175$ & $-0,122^{\star \star \star}$ \\
\hline & & & & & & & & & & $(0,165)$ & $(0,041)$ \\
\hline Constante & $\begin{array}{l}-0,109 \\
(0,077)\end{array}$ & $\begin{array}{l}-0,111 \\
(0,077)\end{array}$ & $\begin{array}{c}-0,116 \\
(0,080)\end{array}$ & $\begin{array}{l}-0,114 \\
(0,083)\end{array}$ & $\begin{array}{l}-0,112 \\
(0,084)\end{array}$ & $\begin{array}{l}-0,075 \\
(0,079)\end{array}$ & $\begin{array}{l}-0,075 \\
(0,087)\end{array}$ & $\begin{array}{c}0,377^{\star \star \star} \\
(0,045)\end{array}$ & $\begin{array}{c}0,261^{\star * *} \\
(0,043)\end{array}$ & $\begin{array}{l}-0,086 \\
(0,080)\end{array}$ & $\begin{array}{c}0,265^{\star \star \star} \\
(0,043)\end{array}$ \\
\hline Efectos Fijos a nivel departamento & $\mathrm{Si}$ & $\mathrm{Si}$ & $\mathrm{Si}$ & $\mathrm{Si}$ & $\mathrm{Si}$ & $\mathrm{Si}$ & $\mathrm{Si}$ & $\mathrm{Si}$ & $\mathrm{Si}$ & $\mathrm{Si}$ & $\mathrm{Si}$ \\
\hline Cantidad de Observaciones & 506 & 506 & 506 & 506 & 506 & 506 & 506 & 506 & 506 & 506 & 506 \\
\hline R2 & 0,003 & 0,004 & 0,004 & 0,004 & 0,004 & 0,003 & 0,005 & 0,642 & 0,659 & 0,006 & 0,660 \\
\hline
\end{tabular}

Errores estándar por clusters entre paréntesis

Nota $1: * * * \mathrm{p}<0,01 ; * * \mathrm{p}<0,05 ; * \mathrm{p}<0,1$.

Nota 2: La variable dependiente en todas las columnas es el cambio en la tasa de mortalidad materna entre el año 2001 y 2004 . El grado de exposición es el cociente entre los establecimiento con convenio relativo a la población del año 2001; la segunda alternativa considera el grado de exposición de un departamento como el propio grado de exposición más la suma ponderada del grado de exposición de los cinco departamentos más cercanos. El cambio poblacional es, valga la redundancia, el cambio poblacional entre 2001 y 2004; lo mismo vale para el cambio en los nacidos vivos. La intensidad inicial es el cociente entre la población y la cantidad de establecimientos públicos de salud en el año 2001 y la tasa de mortalidad materna inicial es la que se corresponde con el período base, es decir con el 2001. Plan Nacer es una variable binaria que identifica aquellos departamentos que se encuentran en las regiones bajo programa.

Nota 3: En todas las regresiones se consideran aquellos departamentos que modifican su tasa de mortalidad maternal como máximo hasta 15 puntos porcentuales en valor absoluto. 
Figura 1.Mortalidad Infantil en Argentina. Período 2000-2009

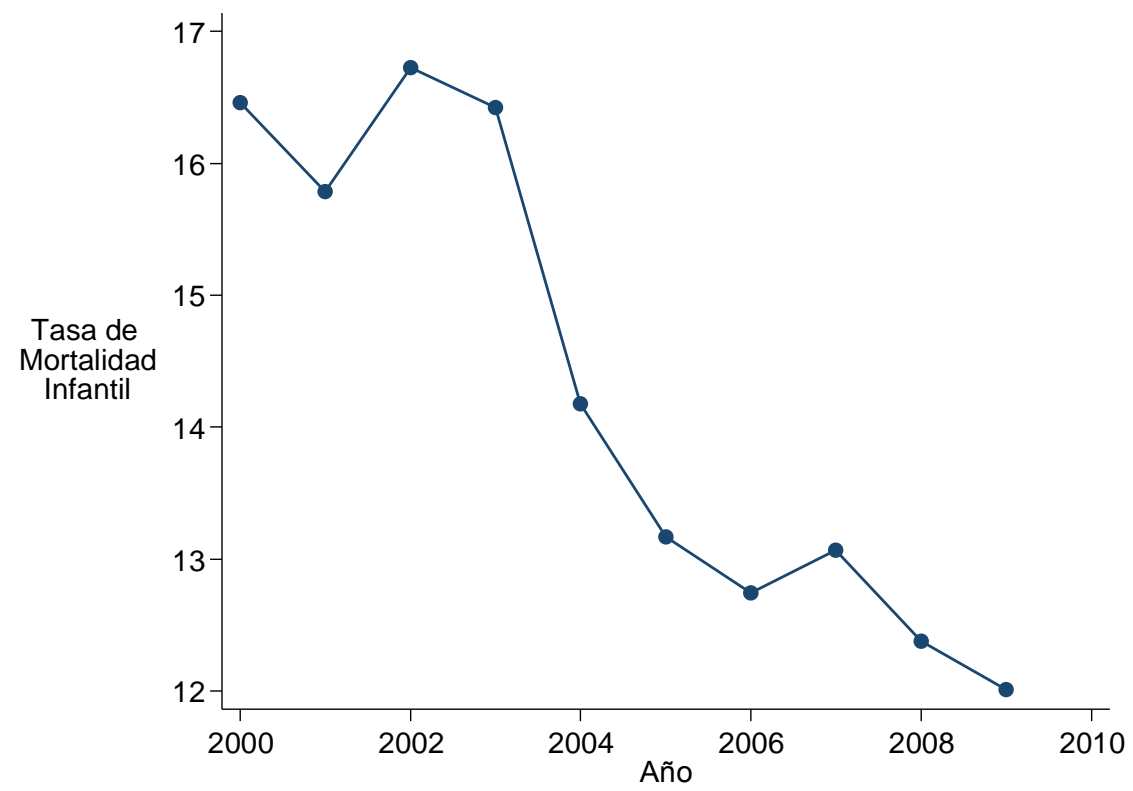

Fuente: Elaboración Propia en base a los Boletines 95, 99, 103, 110, 111, 114, 118, 121, 125 y129. Ministerio de Salud de la Nación Argentina.

\section{Figura 2. Distribución de la Tasa de Mortalidad Infantil en América. Año 2010}

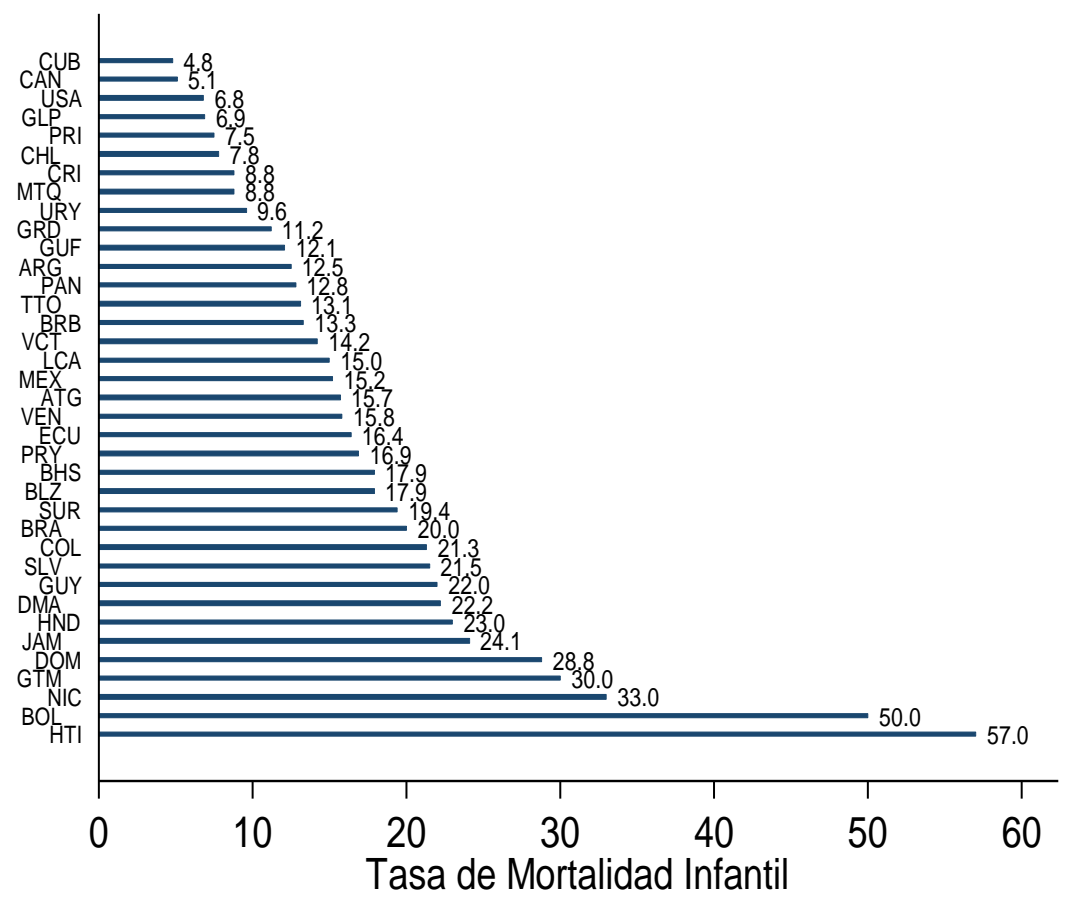

Fuente: Elaboración Propia en base a “Situación de Salud en la Américas. Indicadores Básicos 2010”. 
Figura 3. Evolución de la Mortalidad Infantil en Argentina. Regiones NOA-NEA y resto de Argentina. Período 2000-2004 y 2007

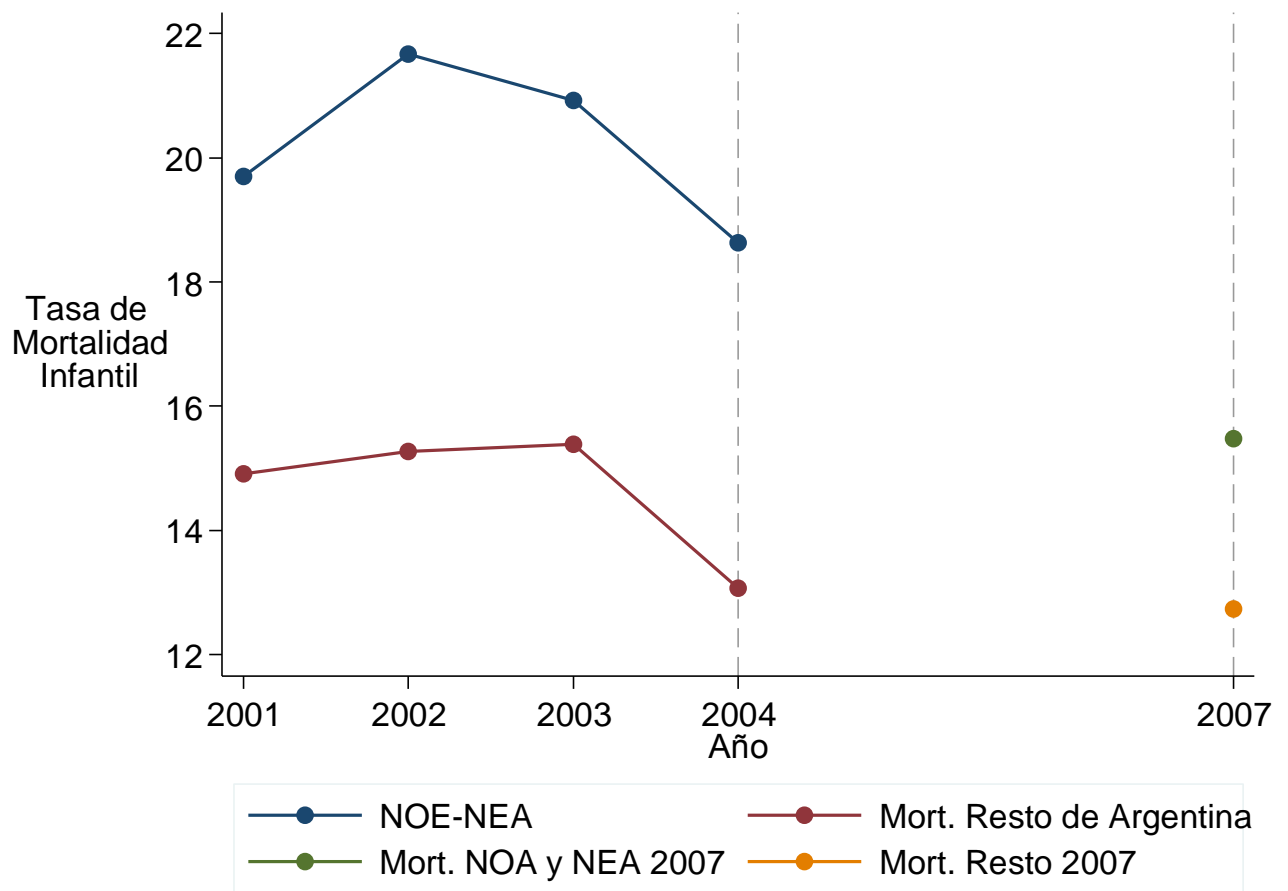

